Two- $f$ I ui $d$ and finite Larmor radi us effects on hi gh- bet a tokanak equil i bri a wi th fl ow i $\mathrm{n}$ r educed nagnet ohydr odynamics

\begin{tabular}{|l|l|}
\hline $\begin{array}{l}\text { j our nal or } \\
\text { publ i cat i on ti tl e }\end{array}$ & Physi ca Scri pt a \\
\hline vol une & 96 \\
\hline page range & 035602 \\
\hline year & 2021- 01- 14 \\
\hline URL & ht t p: //hdl . handl e. net /10655/00012579 \\
\hline
\end{tabular}




\title{
Two-fluid and finite Larmor radius effects on high-beta tokamak equilibria with flow in reduced magnetohydrodynamics
}

\author{
Atsushi Ito ${ }^{1}$ and Noriyoshi Nakajima ${ }^{2}$ \\ ${ }^{1}$ National Institute for Fusion Science, National Institutes of Natural Sciences, 322-6 \\ Oroshi-cho, Toki, Gifu 509-5292 Japan \\ ${ }^{2}$ Rokkasho Research Center, National Institute for Fusion Science, National \\ Institutes of Natural Sciences, 2-166 Omotedate, Obuchi, Rokkasho, Kamikita, \\ Aomori 039-3212 Japan
}

\begin{abstract}
High-beta tokamak equilibria with flow comparable to the poloidal Alfvén velocity in the reduced magnetohydrodynamics (MHD) model with two-fluid and ion finite Larmor radius (FLR) effects are investigated. The reduced form of GradShafranov equation for equilibrium with flow, two-fluid and FLR effects is analytically solved for simple profiles. The dependence of the Shafranov shift for the magnetic axis and the equilibrium limits on the poloidal beta and the poloidal Alfvén Mach number are modified by the two-fluid and FLR effects. In the presence of the diamagnetic drift due to the two-fluid effect, the equilibrium depends on the sign of the $E \times B$ drift velocity. The FLR effect suppresses the large modification due to the two-fluid effect. By constructing magnetic flux coordinates and a local equilibrium model from the analytic solution, the effects of the non-circular property of the magnetic flux surfaces in the poloidal cross-section on the components of the curvature vector is examined in detail. The analytic solution is also used for the benchmark of the numerical code. The numerical solutions with non-uniform pressure, density and temperature profiles show similar behavior to analytic solution.
\end{abstract}




\section{Introduction}

Macroscopic equilibrium and stability of plasmas are described by magnetohydrodynamics (MHD). The MHD model is extended to include small scale effects such as two-fluid and finite Larmor radius (FLR) effects $[1,2,3]$. The equations for such extended MHD models are derived from the kinetic equation by taking fluid moments. The two-fluid effects are the Hall current and electron pressure in Ohm's law. The FLR effects are the gyroviscosity and perpendicular heat fluxes in the fluid moment equations. These effects introduce the scale lengths of the skin depth and the Larmor radius of ions.

An example of MHD stability theory to extend is for the Rayleigh-Taylor (RT) instability (or interchange g-mode) in slab geometry $[3,4,5,6,7]$. For the short wavelength modes of the RT instability, small scale effects cannot be neglected. In the case of constant temperature, the two-fluid and the FLR effects are separately stabilizing [4]. On the other hand, in the case of non-constant temperature, comparison between four models, MHD, two-fluid MHD, MHD with FLR and two-fluid MHD with FLR, shows complicated beta dependence of the combination of the two-fluid and FLR effects [7]. The diamagnetic effects on the stability of toroidal plasmas are studied based on simplified models such as drift models [8, 9] and reduced two-fluid MHD [10, 11].

The small scale effects on equilibrium become important in improved confinement modes of magnetically confined plasmas. Equilibrium flows play important roles such as the suppression of instability and turbulence transport for the formation of the sharp boundary of a well confined region where the scale length becomes small. The diamagnetic term due to two-fluid effects contributes to plasma flow in the high confinement mode (H-mode) plasmas [12]. Equilibrium with flow in the extended MHD models is necessary for the studies of both equilibrium itself and stability.

The reduced MHD equilibrium equations for high-beta tokamaks with flow comparable to the poloidal Alfvén velocity, two-fluid, and FLR effects were derived [13]. The equation for the lowest order of the magnetic flux function forms the GradShafranov (GS) type equation including terms of poloidal $E \times B$ and diamagnetic drifts. In the lowest order, other quantities such as pressure and electrostatic potential are free functions of the magnetic flux. The two-fluid effects introduce the diamagnetic drift to the poloidal flow. The convection due to the diamagnetic drift is canceled by the FLR effect. This is called the gyroviscous cancellation $[1,8,14]$. This GS type equation is a simplified equation of the generalized GS equation for MHD equilibrium with flow [15] and two-fluid equilibrium $[16,17]$, with an extension to include both the two-fluid and FLR effects perturbatively.

In this paper, we solve analytically and numerically the reduced MHD equilibrium equations for high-beta tokamaks with flow comparable to the poloidal Alfvén velocity, two-fluid, and FLR effects. We compare the four models as the study of the RT instability [7] mentioned above. The analytic solution is easily obtained as an extension of the static MHD case $[18,19,20]$ and the case of MHD with flow [21] when the density is constant. This is different from the case of flow comparable to the poloidal 
sound velocity, which is slower than the poloidal Alfvén velocity, where the reduced equilibrium equations have those effects in the next order [22, 23, 24, 25, 26, 27]. Since the GS equation in that order couples with other equations such as pressure equation, those equations with two-fluid and FLR effects are studied numerically [22, 24]. On the other hand, by using the analytic solution, the effects of flow, two-fluid and FLR on the magnetic structure can be examined in detail. From the radial profile of the magnetic flux function, the Shafranov shift for the magnetic axis and the equilibrium limits due to the effects of high-beta and flow are obtained. By constructing magnetic flux coordinates from the analytic solution such those for the static MHD equilibrium [27, 28], the components of the magnetic curvature are analytically obtained. These are expected to be applied to the analysis of the kink, interchange and ballooning instabilities. Analytic representations of flux coordinates and the components of the magnetic curvature enables detailed parameter study of non-circular property of the poloidal cross-sections of the magnetic flux surfaces due to high-beta, flow and diamagnetic effects. We construct a local equilibrium model [38, 39, 40, 41, 42, 43] from the expansion of the analytic solution in the vicinity of the magnetic axis that includes these effects as shaping factors such as the elongation, triangularity and Shafranov shift of the magnetic flux surfaces. The flux surface average of the normal curvature is analytically obtained from the local equilibrium model. The analytic solution can also be used for the benchmark of the numerical code. The numerical code can obtain equilibrium solutions with more complicated profiles.

This paper is organized as follows. In section 2, we introduce the equations for equilibria with flow comparable to the poloidal Alfvén velocity, two-fluid and FLR effects. In section 3, assumptions for analytic and numerical solutions are shown. In section 4, we obtain an analytic solution, magnetic flux coordinates and a local equilibrium model. The parameter dependence of the magnetic structure is examined by comparing between different MHD models. In section 5, numerical solutions are obtained with the finite element method. A summary is given in section 6 .

\section{Reduced two-fluid equilibria with flow comparable to the poloidal Alfvén velocity}

In this section, we briefly introduce the reduced MHD equilibrium equations for highbeta tokamaks with flow comparable to the poloidal Alfvén velocity, two-fluid and FLR effects. The details of the derivation is shown in [13]. The extended MHD equations with two-fluid and ion FLR effects are

$$
\begin{aligned}
& \nabla \cdot(n \mathbf{v})=0, \\
& \nabla \times \mathbf{E}=0, \\
& m_{i} n \mathbf{v} \cdot \nabla \mathbf{v}=\mathbf{j} \times \mathbf{B}-\nabla\left(p_{i}+p_{e}\right)-\lambda_{i} \nabla \cdot \Pi_{i}^{g v}, \\
& \mathbf{E}+\mathbf{v} \times \mathbf{B}=\frac{\lambda_{H}}{n e}\left(\mathbf{j} \times \mathbf{B}-\nabla p_{e}\right),
\end{aligned}
$$




$$
\begin{aligned}
& \mu_{0} \mathbf{j}=\nabla \times \mathbf{B} \\
& \mathbf{v} \cdot \nabla p_{i}+\gamma p_{i} \nabla \cdot \mathbf{v}+\lambda_{i}\left(\frac{2}{5} \gamma \nabla \cdot \mathbf{q}_{i}\right)=0 \\
& \left(\mathbf{v}-\lambda_{H} \mathbf{j} / n e\right) \cdot \nabla p_{e}+\gamma p_{e} \nabla \cdot\left(\mathbf{v}-\lambda_{H} \mathbf{j} / n e\right) \\
& +\lambda_{i}\left(\frac{2}{5} \gamma \nabla \cdot \mathbf{q}_{e}\right)=0
\end{aligned}
$$

where $m_{i}$ is the ion mass, $n$ is the density, $\mathbf{v}$ is the ion flow velocity, $\mathbf{E}$ and $\mathbf{B}$ are the electric and magnetic fields, $\mathbf{j}$ is the current density, $p_{i}$ and $p_{e}$ are the ion and electron pressures, $\Pi_{i}^{g v}$ is the ion gyroviscous tensor, $\mathbf{q}_{i}$ and $\mathbf{q}_{e}$ are the ion and electron heat fluxes, respectively, and $\gamma=5 / 3$. The diagonal components of the pressure tensors are assumed to be isotropic. The explicit forms of $\Pi_{i}^{g v}, \mathbf{q}_{i}$ and $\mathbf{q}_{e}$ used in the derivation are shown below. We have introduced the artificial indices $\lambda_{H}$ and $\lambda_{i}$ that label the two-fluid and FLR terms respectively: $\left(\lambda_{H}, \lambda_{i}\right)=(0,0)$ for the MHD, $(1,0)$ for the two-fluid MHD (TF model), $(0,1)$ for the MHD with FLR (FLR model) and $(1,1)$ for the two-fluid MHD with FLR (FLR+TF model). The electron mass $m_{e}$ is neglected because $m_{e} \ll m_{i}$. The electron gyroviscosity is also neglected since $\rho_{e} \ll \rho_{i}$.

We consider the toroidal axisymmetric equilibria, where, in cylindrical coordinates $(R, \varphi, Z)$, the magnetic field $\mathbf{B}$, the current density $\mathbf{j}$ and the electric field $\mathbf{E}$ can be written as

$$
\begin{aligned}
& \mathbf{B}=\nabla \psi(R, Z) \times \nabla \varphi+I(R, Z) \nabla \varphi \\
& \mu_{0} \mathbf{j}=\nabla I \times \nabla \varphi-\Delta^{*} \psi \nabla \varphi \\
& \mathbf{E}=-\nabla \Phi(R, Z)
\end{aligned}
$$

where $\psi$ is the poloidal magnetic flux and $\Delta^{*} \equiv R^{2} \nabla \cdot\left(R^{-2} \nabla\right)$.

The reduced MHD equilibrium equation is obtained with the asymptotic expansion. The asymptotic expansion is defined in terms of the inverse aspect ratio $\varepsilon \equiv a / R_{0} \ll 1$ where $a$ and $R_{0}$ are the characteristic scale lengths of the minor and major radii respectively. The following high-beta tokamak orderings for reduced MHD [29] are applied,

$$
\begin{aligned}
& B_{p} \sim \varepsilon B, \\
& p_{i} \sim p_{e} \sim \varepsilon\left(B^{2} / \mu_{0}\right), \\
& |\nabla| \sim 1 / a,
\end{aligned}
$$

where $B \equiv \sqrt{B_{p}^{2}+B_{\varphi}^{2}}, B_{p} \equiv|\nabla \psi| / R$ is the poloidal magnetic field and $B_{\varphi} \equiv I / R$ is the toroidal magnetic field. We consider the case of flow comparable to the poloidal Alfvén velocity, $v \equiv|\mathbf{v}| \sim V_{A p} \equiv B_{p} /\left(\mu_{0} m_{i} n\right)^{1 / 2}$,

$$
m_{i} n v^{2} \sim\left\|\Pi_{i}^{g v}\right\| \sim \varepsilon p \sim \varepsilon^{2} B^{2} / \mu_{0},
$$

and we assume

$$
v \sim \frac{|\mathbf{j}|}{n e} \sim \frac{|\nabla p|}{n e B},
$$




$$
\left|\mathbf{q}_{i, e}\right| \sim v p_{i, e} .
$$

The two-fluid and FLR effects have the scale lengths of the ion skin depth $d_{i}$ and ion Larmor radius $\rho_{i}$, respectively,

$$
\begin{aligned}
& d_{i}=\sqrt{\frac{m_{i}}{\mu_{0} e^{2} n}}, \\
& \rho_{i}=d_{i} \sqrt{\frac{p_{i}}{B^{2} / \mu_{0}}} .
\end{aligned}
$$

To be consistent with the slow dynamics (drift) ordering $[2,8]$ for the reduced MHD models with flow comparable to the poloidal Alfvén velocity, the orderings for the scale lengths $d_{i} / a \sim 1$ and $\rho_{i} / a \sim \sqrt{\varepsilon}$ are required. This is the standard ordering for reduced two-fluid MHD [10, 11]. The variables are expanded in orders of $\varepsilon$ as

$$
\begin{aligned}
& \psi=\psi_{1}+\psi_{2}+\ldots, \\
& I=B_{0} R_{0}+I_{1}+I_{2}+\ldots, \\
& p_{i}=p_{i 1}+p_{i 2}+\ldots, \\
& p_{e}=p_{e 1}+p_{e 2}+\ldots, \\
& n=n_{0}+\ldots, \\
& \Phi=\Phi_{1}+\ldots, \\
& R=R_{0}+x,
\end{aligned}
$$

where $\psi_{j} \sim \varepsilon^{j} a R_{0} B_{0}, p_{\{i, e\} j} \sim \varepsilon^{j} B_{0}^{2} / \mu_{0}(j=1,2 \ldots)$ and $B_{0}$ and $R_{0}$ are constant. The leading order of the force balance equation (3) yields

$$
p_{i 1}+p_{e 1}+\frac{B_{0}}{\mu_{0} R_{0}} I_{1}=\text { const }
$$

The ion flow velocity $\mathbf{v}$ is separated as

$$
\begin{aligned}
& \mathbf{v} \equiv \mathbf{v}_{E}+\lambda_{H} \mathbf{v}_{d i}+v_{\|} R_{0} \nabla \varphi, \\
& \mathbf{v}_{E} \simeq-B_{0}^{-1} \nabla \Phi_{1} \times\left(R_{0} \nabla \varphi\right), \\
& \mathbf{v}_{d i} \simeq-\frac{1}{e B_{0} n_{0}} \nabla p_{i 1} \times\left(R_{0} \nabla \varphi\right),
\end{aligned}
$$

where $\mathbf{v}_{E}$ and $\mathbf{v}_{d i}$ are the $E \times B$ and ion diamagnetic drift velocities, respectively, and $v_{\|}$ is the parallel flow velocity, all of which are comparable to the poloidal Alfvén velocity. The ion gyroviscous force and heat fluxes in $[2,31]$ are needed only their leading orders,

$$
\begin{aligned}
& \nabla \cdot \Pi_{i}^{g v} \simeq-m_{i} n \mathbf{v}_{d i} \cdot \nabla \mathbf{v}-\nabla\left(\chi_{v}+\chi_{q}\right), \\
& \chi_{v} \equiv \frac{m_{i} p_{i}}{2 e B^{2}} \mathbf{B} \cdot(\nabla \times \mathbf{v}), \\
& \chi_{q} \equiv \frac{m_{i}}{5 e B^{2}} \mathbf{B} \cdot\left(\nabla \times \mathbf{q}_{i \perp}\right) . \\
& \mathbf{q}_{i} \simeq \mathbf{q}_{i \perp} \simeq \frac{5}{2} \frac{1}{e B^{2}} \mathbf{B} \times\left[p_{i} \nabla\left(\frac{p_{i}}{n}\right)\right],
\end{aligned}
$$




$$
\mathbf{q}_{e} \simeq \mathbf{q}_{e \perp} \simeq-\frac{5}{2} \frac{1}{e B^{2}} \mathbf{B} \times\left[p_{e} \nabla\left(\frac{p_{e}}{n}\right)\right]
$$

where $\mathbf{q}_{i \perp}$ and $\mathbf{q}_{e \perp}$ are the ion and electron diamagnetic perpendicular heat fluxes respectively. Substituting (20) and (23), (3) becomes

$$
\begin{aligned}
& m_{i} n\left[\mathbf{v}_{E}+\left(\lambda_{H}-\lambda_{i}\right) \mathbf{v}_{d i}+v_{\|} R_{0} \nabla \varphi\right] \cdot \nabla \mathbf{v} \\
\simeq & \mathbf{j} \times \mathbf{B}-\nabla\left(p_{i}+p_{e}\right)+\lambda_{i} \nabla\left(\chi_{v}+\chi_{q}\right) .
\end{aligned}
$$

The left hand side of (28) shows that the convection due to the diamagnetic drift vanishes for the FLR+TF model, $\left(\lambda_{H}, \lambda_{i}\right)=(1,1)$. This is the FLR effect on the convective terms that appear from the gyroviscous tensor $\Pi_{i}^{g v}$, known as the gyroviscous cancellation $[1,8,14]$. The gyroviscous cancellation in (28) is of the type of [14]. It is shown that the following quantities are the functions of $\psi_{1}, n_{0}\left(\psi_{1}\right), \Phi_{0}\left(\psi_{1}\right), p_{i 1}\left(\psi_{1}\right), p_{e 1}\left(\psi_{1}\right), v_{\|}\left(\psi_{1}\right)$ and $I_{1}\left(\psi_{1}\right)$. The following relation for the second order quantities are obtained from the poloidal component of the force balance equation (28) as

$$
\begin{aligned}
& p_{i 2}+p_{e 2}+\frac{B_{0}}{\mu_{0} R_{0}} I_{2} \\
& +\frac{m_{i} n_{0}}{B_{0}^{2}}\left[\Phi_{1}^{\prime}+\frac{\left(\lambda_{H}-\lambda_{i}\right) p_{i 1}^{\prime}}{e n_{0}}\right]\left(\Phi_{1}^{\prime}+\frac{\lambda_{H} p_{i 1}^{\prime}}{e n_{0}}\right) \frac{\left|\nabla \psi_{1}\right|^{2}}{2} \\
& -\lambda_{i}\left(\chi_{v}+\chi_{q}\right) \equiv g_{*}\left(\psi_{1}\right),
\end{aligned}
$$

where the prime denotes the derivative with respect to $\psi_{1}$. The reduced Grad-Shafranov (GS) equation with flow, two-fluid and ion FLR effects is obtained from the radial component of the force balance equation (28) as

$$
\begin{aligned}
& {\left[1-F\left(\psi_{1}\right)\right]\left(\frac{\partial^{2}}{\partial R^{2}}+\frac{\partial^{2}}{\partial Z^{2}}\right) \psi_{1}-\frac{\left|\nabla \psi_{1}\right|^{2}}{2} F^{\prime}\left(\psi_{1}\right)} \\
& =-\mu_{0} R_{0}^{2}\left(\frac{2 x}{R_{0}} p_{1}^{\prime}+g_{*}^{\prime}\right)-\left(\frac{I_{1}^{2}}{2}\right)^{\prime},
\end{aligned}
$$

where $p_{1} \equiv p_{i 1}+p_{e 1}$,

$$
\begin{aligned}
F\left(\psi_{1}\right) & \equiv\left(\frac{V_{E}}{V_{A p}}+\lambda_{H} \frac{V_{d i}}{V_{A p}}\right)\left[\frac{V_{E}}{V_{A p}}+\left(\lambda_{H}-\lambda_{i}\right) \frac{V_{d i}}{V_{A p}}\right], \\
\frac{V_{E}}{V_{A p}} & \equiv-\sqrt{\mu_{0} m_{i} n_{0}} \frac{R_{0} \Phi_{1}^{\prime}}{B_{0}}, \\
\frac{V_{d i}}{V_{A p}} & \equiv-\sqrt{\mu_{0} m_{i} n_{0}} \frac{R_{0} p_{i 1}^{\prime}}{e n_{0} B_{0}} .
\end{aligned}
$$

Equation (31) represents the contribution of the convective term. Equations (32) and (33) are the poloidal Alfvén Mach numbers of the poloidal flow velocities of the $E \times B$ and ion diamagnetic drifts, respectively. The parallel flow $v_{\|}$does not appear in (30) by the ordering. The parallel heat flux is also ordered out. The gyroviscous cancellation, $\lambda_{H}-\lambda_{i}=0$, occurs in (31) for the FLR+TF model, $\left(\lambda_{H}, \lambda_{i}\right)=(1,1)$. The FLR+TF model is consistent with the reduced two-fluid MHD system with time evolution in [11]. It is noted that the present model does not reproduce the resolution of the Alfvén singularity, $F=1$, by the Hall current as in non-reduced two-fluid models [30]. Thus, equation (30) is valid when $|1-F| \sim O(1)$. 


\section{Assumptions for analytic and numerical solutions}

To solve the reduced GS equation (30) analytically and numerically, we adopt quasitoroidal coordinates $(r, \theta, \phi)$ where $R=R_{0}+r \cos \theta$ and $Z=r \sin \theta$. We then apply the following normalization,

$$
\begin{aligned}
& r / a \equiv \bar{r}, \quad \psi_{1} / \psi_{c} \equiv \bar{\psi}_{1}, \quad \psi_{2} / \psi_{c} \equiv \varepsilon \bar{\psi}_{2} \\
& \psi_{c} / B_{0} R_{0} a \equiv \varepsilon B_{p c}, \quad x / a \equiv \bar{x}, \quad Z / a \equiv \bar{z} .
\end{aligned}
$$

The quantities with the subscript "c" are the non-dimensional constant characteristic quantities. The fixed boundary conditions for $\bar{\psi}_{1}$ at circular boundary is assumed,

$$
\bar{\psi}_{1}(\bar{r}=1, \theta)=0 .
$$

Here, we assume the profiles of the ion and electron pressures as,

$$
p_{\{i, e\} 1}=\varepsilon\left(B_{0}^{2} / \mu_{0}\right) p_{\{i, e\} 1 c} \bar{\psi}_{1}^{\alpha} .
$$

We assume the profile of $n_{0}$ as,

$$
n_{0}=n_{0 c} \bar{\psi}_{1}^{\kappa} \text {. }
$$

The poloidal Alfvén Mach number of the poloidal velocity of the $E \times B$ drift, (32), is assumed to be constant,

$$
V_{E} / V_{A p}=V_{E c} .
$$

The poloidal Alfvén Mach number of the poloidal velocity of the diamagnetic drift, (33), is given by

$$
V_{d i} / V_{A p}=\alpha V_{d c} p_{i 1 c} \bar{\psi}_{1}^{\alpha-1-\kappa / 2}
$$

where

$$
V_{d c} \equiv-\frac{1}{B_{p c} a} \sqrt{\frac{m_{i}}{\mu_{0} e^{2} n_{0 c}}} .
$$

In an analogy to the MHD [21], we assume

$$
g_{*}+\frac{I_{1}^{2}}{2 \mu_{0} R_{0}^{2}}=\varepsilon^{2}\left(B_{0}^{2} / \mu_{0}\right)(1-F) g_{c} \bar{\psi}_{1} .
$$

The convective term $F$, (31), is given by

$$
\begin{aligned}
F= & \left(V_{E c}+\lambda_{H} \alpha V_{d c} p_{i 1 c} \bar{\psi}_{1}^{\alpha-1-\kappa / 2}\right) \\
& \times\left[V_{E c}+\left(\lambda_{H}-\lambda_{i}\right) \alpha V_{d c} p_{i 1 c} \bar{\psi}_{1}^{\alpha-1-\kappa / 2}\right] .
\end{aligned}
$$

We examine the cases where $F=$ const., i.e., both of the poloidal Alfvén Mach numbers of the $E \times B$ and diamagnetic poloidal flow velocities are constant,

$$
\alpha-1-\kappa / 2=0 .
$$

The GS equation (30) is rewritten as

$$
\left[\frac{1}{\bar{r}} \frac{\partial}{\partial \bar{r}}\left(\bar{r} \frac{\partial}{\partial \bar{r}}\right)+\frac{1}{\bar{r}^{2}} \frac{\partial^{2}}{\partial \theta^{2}}\right] \bar{\psi}_{1}=-\frac{g_{c}}{B_{p c}^{2}}-\frac{2 \alpha p_{1 c} \bar{\psi}_{1}^{\alpha-1} \bar{r} \cos \theta}{(1-F) B_{p c}^{2}},
$$




$$
F=\left(V_{E c}+\lambda_{H} \alpha V_{d c} p_{i 1 c}\right)\left[V_{E c}+\left(\lambda_{H}-\lambda_{i}\right) \alpha V_{d c} p_{i 1 c}\right]
$$

where $p_{1 c} \equiv p_{i 1 c}+p_{e 1 c}$. We consider the case where the poloidal Alfvén Mach number of the poloidal flow velocity of the diamagnetic drift is relatively small, $V_{d c}=-0.2$, $g_{c}=2.5, B_{p c}=1.0$ and $p_{i 1 c}=p_{e 1 c}=p_{1 c} / 2$.

\section{Parameter dependence of analytic solution}

\subsection{Analytic solution}

An analytic solution for (43) can be found when the pressure profile is linear and the density is constant, $\alpha=1$ and $\kappa=0$. In this case, (43) and (44) becomes

$$
\begin{aligned}
& {\left[\frac{1}{\bar{r}} \frac{\partial}{\partial \bar{r}}\left(\bar{r} \frac{\partial}{\partial \bar{r}}\right)+\frac{1}{\bar{r}^{2}} \frac{\partial^{2}}{\partial \theta^{2}}\right] \bar{\psi}_{1}=-\frac{g_{c}}{B_{p c}^{2}}-\frac{2 p_{1 c} \bar{r} \cos \theta}{(1-F) B_{p c}^{2}},} \\
& F=\left(V_{E c}+\lambda_{H} V_{d c} p_{i 1 c}\right)\left[V_{E c}+\left(\lambda_{H}-\lambda_{i}\right) V_{d c} p_{i 1 c}\right] .
\end{aligned}
$$

Equation (45) is solved with the boundary condition (34) by

$$
\bar{\psi}_{1}=\frac{1-\bar{r}^{2}}{4 B_{p c}^{2}}\left(g_{c}+\frac{p_{1 c} \bar{r} \cos \theta}{1-F}\right) .
$$

For the MHD, the analytic solution (47) coincides with that of [21]. For the static MHD equilibrium [18, 19], $F=0$, the magnetic axis is shifted outwards in a torus due to the Shafranov shift and the poloidal cross-section of magnetic flux surfaces become non-circular due to high-beta. The Alfvén singularity, $F=1$, occurs when

$$
V_{E c}=\frac{1}{2}\left[-\left(2 \lambda_{H}-\lambda_{i}\right) V_{d c} p_{i 1 c} \pm \sqrt{\left(\lambda_{i} V_{d c} p_{i 1 c}\right)^{2}+4}\right] \text {. }
$$

We examine the parameter dependence of the effects of flow, two-fluid and FLR on the magnetic structure of the analytic solution in the rest of this section.

The Shafranov shift for the magnetic axis, $(\bar{x}, \bar{z})=\left(\bar{\Delta}_{s}, 0\right)$, from the geometric center, $(\bar{x}, \bar{z})=(0,0)$, is obtained from

$$
\left.\frac{d \bar{\psi}_{1}(\bar{x}, \bar{z}=0)}{d \bar{x}}\right|_{\bar{x}=\bar{\Delta}_{s}}=0
$$

which yields

$$
\bar{\Delta}_{s}=\frac{-1+\sqrt{1+3[\nu /(1-F)]^{2}}}{3 \nu /(1-F)},
$$

where $\nu \equiv p_{1 c} / g_{c}$, and $\nu / \varepsilon$ represents the poloidal beta for $F=0$ [19]. Figure 1 (a) shows the dependence of $\bar{\Delta}_{s}$ on the poloidal Alfvén Mach number of the $E \times B$ poloidal flow velocity $V_{E c}$ for different models for $\nu=0.8$. Figure 1 (b) shows the behavior of $\bar{\Delta}_{s}$ for the region around $V_{E c}=0$. For the MHD, the Shafranov shift $\bar{\Delta}_{s}$ is symmetric with respect to the sign of $V_{E c}$. The Shafranov shift $\bar{\Delta}_{s}$ for the MHD increases with $\left|V_{E c}\right|$ for sub-Alfvénic poloidal flow, $\left|V_{E c}\right|<1$, and become negative for super-Alfvénic poloidal flow, $\left|V_{E c}\right|>1$, across the Alfvén singularity at $\left|V_{E c}\right|=1$ [21]. For the other three 
models, $\bar{\Delta}_{s}$ is asymmetric with respect to the sign of $V_{E c}$ due to the diamagnetic flow. The Alfvén singularity appears when $V_{E c}$ satisfies (48). The TF model shows large shift from the MHD. Since the poloidal flow in the TF model is the sum of the $E \times B$ and the ion diamagnetic drifts, the curve of the TF model is shifted from that of the MHD by $-V_{d c} p_{i 1 c}$ in the horizontal direction. The effect of flow is enhanced (suppressed) when the signs of the $E \times B$ and the ion diamagnetic drifts are the same (opposite). For the FLR+TF model, the large shift due to the two-fluid effect is suppressed due to the gyroviscous cancellation except for the region around $V_{E c}=0$. For the FLR model, the asymmetry is opposite to that of the FLR+TF model. Figure 1 (b) shows that the FLR and FLR+TF models do not indicate the simple horizontal shift from the MHD and that the MHD, FLR and FLR+TF models coincide with each other at $V_{E c}=0$.

Figure 2 shows the radial profiles of $\bar{\psi}_{1}$ in the midplane, $\bar{z}=0$, for different models for super-Alfvénic poloidal $E \times B$ flow $\left|V_{E c}\right|=1.5$, for $\nu=0.8$. For all of the four models, the magnetic axis, the peak of $\bar{\psi}_{1}$, is located in $\bar{x}<0$ as shown in figure 1 . For the TF model, the radial profile is significantly modified from that for the MHD and the separatrix, the minimum of $\bar{\psi}_{1}$, appears in the plasma region for $V_{E c}=1.5$. Since the pressure $p_{1}$ becomes negative at the separatrix, this equilibrium is not realistic. The condition that the separatrix does not enter into the plasma region gives the equilibrium beta limit $[19,21]$, which is obtained from

$$
\left.\frac{\partial \bar{\psi}_{1}}{\partial \bar{r}}\right|_{\bar{r}=1}=-\frac{g_{c}}{2 B_{p c}^{2}}\left(1+\frac{\nu}{1-F} \cos \theta\right)<0
$$

for $-\pi \leq \theta \leq \pi$. When $F=0$, the beta limit $\nu<1$ for static MHD equilibrium [19] is reproduced. When $F<1$ that corresponds to the sub-Alfvénic poloidal flow, the equilibrium beta limit is $\nu<1-F$ which yields the following limits of the $E \times B$ poloidal flow for a fixed $\nu$,

$$
\begin{aligned}
& V_{E c 1-}<V_{E c}<V_{E c 1+}, \\
& V_{E c 1 \pm}=\frac{1}{2}\left[-\left(2 \lambda_{H}-\lambda_{i}\right) V_{d c} p_{i 1 c} \pm \sqrt{\left(\lambda_{i} V_{d c} p_{i 1 c}\right)^{2}+4(1-\nu)}\right] .
\end{aligned}
$$

Analogously, when $F>1$ that corresponds to the super-Alfvénic poloidal flow, the equilibrium beta limit is $\nu<F-1$ which yields the following limits of the $E \times B$ poloidal flow for a fixed $\nu$,

$$
\begin{aligned}
& V_{E c}<V_{E c 2-} \quad \text { or } \quad V_{E c}>V_{E c 2+}, \\
& V_{E c 2 \pm}=\frac{1}{2}\left[-\left(2 \lambda_{H}-\lambda_{i}\right) V_{d c} p_{i 1 c} \pm \sqrt{\left(\lambda_{i} V_{d c} p_{i 1 c}\right)^{2}+4(1+\nu)}\right] .
\end{aligned}
$$

Figure 3 shows the limits of the $E \times B$ poloidal flow, (52) and (54), on the contour map of the Shafranov shift $\bar{\Delta}_{s}$ in the $V_{E c}-\nu$ plane. The dependence of $\bar{\Delta}_{s}$ on $V_{E c}$ at $\nu=0.8$ is equivalent to figure 1. The forbidden regions are $V_{E c 2-} \leq V_{E c} \leq V_{E c 1-}$ and $V_{E c 1+} \leq V_{E c} \leq V_{E c 2+}$, which are located around the Alfvén singularity. The vertical direction shows the beta limit for a fixed $V_{E c}$. Figure 3 (a) shows the result for the MHD. 
It is symmetric with respect to the sign of $V_{E c}$. The beta limit $\nu<1$ for static MHD equilibrium corresponds to the vertical line at $V_{E c}=0$. The dependence of $\bar{\Delta}_{s}$ on $\left|V_{E c}\right|$ with a forbidden region at some $\nu(<1)$ corresponds to figure 3 of [21]. The amplitude of the Shafranov shift increases and the forbidden regions become large with an increase of the poloidal beta; $\nu / \varepsilon$. As the $\left|V_{E c}\right|$ increases, the limit of $\nu$ decreases for sub-Alfvénic poloidal flow, $\left|V_{E c}\right|<1$, and increases for super-Alfvénic poloidal flow, $\left|V_{E c}\right|>1$. For $\left|V_{E c}\right|^{2} \geq 2, \nu \geq 1$ is allowed. Figure 3 (b) shows the result for the TF model. The $V_{E c}$ dependencies of the Shafranov shift are shifted by $-V_{d c} p_{i 1 c}$ for a fixed $\nu$. Since $p_{i 1 c}$ is proportional to $\nu$ from the definition, the shift is also proportional to $\nu$. The parameters for figure $2,\left(V_{E c}, \nu\right)=(1.5,0.8)$, is in the forbidden region $V_{E c 1+} \leq V_{E c} \leq V_{E c 2+}$ only for the TF model. Figures 3 (c) and (d) show the results for the FLR and FLR+TF models. Compared to the TF model, the results of FLR and FLR+TF models show moderate shifts except for the region around $V_{E c}=0$. The shifts of the FLR and FLR+TF models from the MHD are opposite to each other. There are two points in $V_{E c}$ where $F$ becomes zero, $V_{E c}=0, V_{d c} p_{i 1 c}$ for the FLR model and $V_{E c}=0,-V_{d c} p_{i 1 c}$ for the FLR+TF model. Between these two points, there is a region where $\nu \geq 1$ occurs within the limit, in addition to those regions for super-Alfvénic poloidal flow.

\subsection{Magnetic flux coordinates}

The magnetic flux coordinates $(\xi, \Theta)$ in the poloidal cross-section are obtained from the analytic solution of $\psi_{1},(47)$, by the relations, the same as those for the static MHD equilibrium [28, 27],

$$
\begin{aligned}
& \left(1-\xi^{2} \cos ^{2} \Theta\right) \bar{\psi}_{11}\left(\bar{\Delta}_{s}\right)=\bar{\psi}_{11}(\bar{x}), \\
& \left(-\xi^{2} \sin ^{2} \Theta\right) \bar{\psi}_{11}\left(\bar{\Delta}_{s}\right)=\bar{\psi}_{12}(\bar{x}, \bar{z}),
\end{aligned}
$$

where $\bar{\psi}_{1}$ has been rewritten with the Shafranov shift $\bar{\Delta}_{s},(50)$, as

$$
\begin{aligned}
& \bar{\psi}_{1}(\bar{x}, \bar{z})=\bar{\psi}_{11}(\bar{x})+\bar{\psi}_{12}(\bar{x}, \bar{z}), \\
& \bar{\psi}_{11}(\bar{x})=\frac{g_{c}}{4 B_{p}^{2}} \frac{1-3 \bar{\Delta}_{s}^{2}+2 \bar{\Delta}_{s} \bar{x}}{1-3 \bar{\Delta}_{s}^{2}}\left(1-\bar{x}^{2}\right), \\
& \bar{\psi}_{12}(\bar{x}, \bar{z})=-\frac{g_{c}}{4 B_{p}^{2}} \frac{1-3 \bar{\Delta}_{s}^{2}+2 \bar{\Delta}_{s} \bar{x}}{1-3 \bar{\Delta}_{s}^{2}} \bar{z}^{2} .
\end{aligned}
$$

Equations (56) and (57) are analytically solved for $(\bar{x}, \bar{z})$ as

$$
\begin{aligned}
\bar{x} & =\bar{\Delta}_{s}+\frac{1+3 \bar{\Delta}_{s}^{2}}{6 \bar{\Delta}_{s}}\{-1 \\
& \left.+2 \cos \left\{\frac{2}{3} \arccos \left[\frac{3 \sqrt{3} \bar{\Delta}_{s}\left(1-\bar{\Delta}_{s}^{2}\right)}{\left(1+3 \bar{\Delta}_{s}^{2}\right)^{3 / 2}} \xi \cos \Theta\right]\right\}\right\}, \\
\bar{z} & =\frac{1-\bar{\Delta}_{s}^{2}}{\sqrt{1-3 \bar{\Delta}_{s}^{2}+2 \bar{\Delta}_{s} \bar{x}}} \xi \sin \Theta .
\end{aligned}
$$


The magnetic flux coordinates $(\xi, \Theta)$ constitute a non-orthogonal coordinate system in the poloidal cross-section. Figures 4 and 5 show the magnetic flux coordinates for equilibria with sub- and super-Alfvénic poloidal $E \times B$ flow $\left|V_{E c}\right|=0.25$ and 1.5 respectively, for the MHD and FLR+TF models for $\nu=0.8$. The magnetic flux coordinates for the static MHD equilibrium $\left(V_{E c}=0\right)$ is also shown in figure 4 for comparison. It is noted that, in figures $4-14$, only the FLR+TF model is compared with the MHD model since the magnetic flux coordinates cannot be obtained for the TF model for $V_{E c}=1.5$ and the FLR model is equivalent to the opposite sign of $V_{E c}$ of the FLR+TF model. The $\xi$ coordinate represents the magnetic flux surfaces and ranges from $\xi=0$ at the magnetic axis, $(\bar{x}, \bar{z})=\left(\bar{\Delta}_{s}, 0\right)$ to $\xi=1$ at the boundary, $\bar{r}=1$. The $\Theta$ coordinate represents the poloidal angle where $\Theta=0$ for the outer midplane, $\Theta= \pm \pi / 2$ at $\bar{x}=\bar{\Delta}_{s}, \Theta= \pm \pi$ for the inner midplane, $-\pi<\Theta<0$ for the lower half and $0<\Theta<\pi$ for the upper half. For the MHD, the Shafranov shift is slightly enhanced due to sub-Alfvénic poloidal flow (figure 4) while the magnetic axis is located in negative $\bar{x}$ for super-Alfvénic poloidal flow (figure 5). The magnetic flux coordinates are modified due to flow along with the change of the magnetic structure. By including both two-fluid and FLR effects, the magnetic flux coordinates are modified due to the diamagnetic flow and become asymmetric with respect to the sign of $V_{E c}$ (figures 4 and 5).

The magnetic curvature vector is defined as

$$
\boldsymbol{\kappa}=\mathbf{b} \cdot(\nabla \mathbf{b}) \text {, }
$$

where

$$
\mathbf{b}=\mathbf{B} / B
$$

By exploiting the magnetic flux coordinates $(\xi, \Theta)$, the components of the magnetic curvature are obtained. The definitions of the components are based on [32]. The poloidal curvature of magnetic flux surfaces is obtained as

$$
\kappa_{p}=-\mathbf{t} \cdot(\nabla \mathbf{t}) \cdot \mathbf{n}
$$

where

$$
\mathbf{n} \equiv \frac{\nabla \xi}{|\nabla \xi|}, \quad \mathbf{t} \equiv \mathbf{e}_{\varphi} \times \mathbf{n}, \quad \mathbf{e}_{\varphi} \equiv \frac{\nabla \varphi}{|\nabla \varphi|}
$$

The toroidal curvature of the magnetic flux surfaces is given by

$$
\kappa_{\varphi}=-\mathbf{e}_{\varphi} \cdot\left(\nabla \mathbf{e}_{\varphi}\right) \cdot \mathbf{n}
$$

The normal curvature is given by

$$
\kappa_{n}=\mathbf{n} \cdot \boldsymbol{\kappa}
$$

which is rewritten with $\kappa_{p}$ and $\kappa_{\varphi}$ as

$$
\kappa_{n}=-\left(\frac{B_{p}}{B}\right)^{2} \kappa_{p}-\left(\frac{B_{\varphi}}{B}\right)^{2} \kappa_{\varphi} .
$$


In the leading order, the normal curvature $\kappa_{n}$ is dominated by the negative of the toroidal curvature,

$$
\kappa_{n} \simeq-\kappa_{\varphi} .
$$

In the reduced MHD, only the leading order of the normal curvature is taken into account. The geodesic curvature is

$$
\kappa_{g}=\pi \cdot \kappa,
$$

where

$$
\boldsymbol{\pi}=\mathbf{b} \times \mathbf{n} .
$$

The leading order terms of $\kappa_{n}$ and $\kappa_{g}$ arise due to toroidicity. In Appendix A, explicit forms of the components of the magnetic curvature normalized with $a^{-1}, \bar{\kappa}_{p}, \bar{\kappa}_{\varphi}, \bar{\kappa}_{n}$ and $\bar{\kappa}_{g}$, to be analytically obtained with (61) and (62) for $\vartheta=\Theta$ are shown.

Figures 6 and 7 show the poloidal profiles of (a) the poloidal curvature $\bar{\kappa}_{p}$, (b) the normal curvature $\bar{\kappa}_{n}$, (c) the geodesic curvature $\bar{\kappa}_{g}$ and (d) 2D poloidal Jacobian $\bar{D}$, defined in (A.8), at $\xi=0.9$ for the MHD and FLR+TF models with sub- and superAlfvénic poloidal $E \times B$ flow $\left|V_{E c}\right|=0.25$ and 1.5, respectively, for $\nu=0.8$. The results for the static MHD equilibrium are also shown in figure 6 for comparison.

In figure 6 (a), the poloidal curvature for the static MHD equilibrium departs from a constant value $1 / \xi$ due to the high-beta effect. The poloidal curvature depends on the poloidal angle $\Theta$ when the poloidal cross-section of the magnetic flux surface is noncircular [33]. The poloidal curvature for the static MHD equilibrium also indicates the feature of the D shape that the maxima are located inside of the torus, $\pi / 2<|\Theta| \leq \pi$, and the minimum in the inner midplane, $|\Theta|=\pi$, is smaller than that in the outer midplane, $\Theta=0$. The poloidal curvature for the sub-Alfvénic poloidal $E \times B$ flow for the MHD model indicates that the flow enhances the oscillation amplitude of that of the static MHD. On the other hand, in figure 7 (a), the poloidal curvature for the super-Alfvénic poloidal flow indicates the feature of the reversed $\mathrm{D}$ shape that the maxima are located outside of the torus, $0 \leq|\Theta|<\pi / 2$, and the minimum in the inner midplane, $|\Theta|=\pi$, is larger than that in the outer midplane, $\Theta=0$. While the poloidal curvature is symmetric with respect to the sign of $V_{E c}$ for the MHD, it is asymmetric for the FLR+TF model. The effect of non-circularity of the poloidal crosssection of the magnetic flux surfaces on the kink mode was studied in [34, 35, 36, 37]. These results can be applied to the study of the kink instability. In section 4.3, we approximately derive the elongation and triangularity to analyse the non-circularity of the poloidal cross-section of the magnetic surfaces in more detail.

In figure 6 (b), the normal curvature for the static MHD equilibrium departs from the $-\varepsilon \cos \Theta$ curve and the peak width around the outer midplane, $\Theta=0$, becomes broader as the poloidal beta increases. The region for $\bar{\kappa}_{n}<0$ corresponds to the bad-curvature region that drives interchange and ballooning modes. For the normal curvature for the sub-Alfvénic poloidal $E \times B$ flow, the modification due to the highbeta effect is slightly enhanced. In figure 7 (b), for the normal curvature for the superAlfvénic poloidal $E \times B$ flow, the peak width around the outer midplane, $\Theta=0$, 
becomes narrower than that of the $-\varepsilon \cos \Theta$ curve. In both of the figures 6 (b) and 7 (b), for the FLR+TF model, the effects of two-fluid and FLR are small but the normal curvature is asymmetric with respect to the sign of $V_{E c}$. The flux surface average of the normal curvature, defined in (A.9), is needed for the analysis of the interchange and ballooning instabilities. In this coordinate system, the flux surface average of the normal curvature is obtained by numerical integration with the 2D poloidal Jacobian, figures $6(\mathrm{~d})$ and 7 (d) that deviate from $(2 \pi)^{-1}$ due to the high beta effect. In section 4.3, we approximately derive the flux surface average of the normal curvature by using the local equilibrium model.

In figure 6 (c), the geodesic curvature for the static MHD equilibrium deviates from $\varepsilon \sin \Theta$ curve and the maximum and the minimum are shifted from $|\Theta|=\pi / 2$ to inside of the torus, $|\Theta|>\pi / 2$, due to the high-beta effect. For the geodesic curvature $\bar{\kappa}_{g}$ for the sub-Alfvénic poloidal $E \times B$ flow, the modification due to the high-beta effect is slightly enhanced. In figure 7 (c), for the geodesic curvature for the super-Alfvénic poloidal $E \times B$ flow, the maximum and minimum are shifted to outside of the torus, $|\Theta|<\pi / 2$, in contrast to those of the static MHD equilibrium. In both of the figures 6 (c) and 7 (c), for the FLR+TF model, the effects of two-fluid and FLR are small but the normal curvature is asymmetric with respect to the sign of $V_{E c}$. The geodesic curvature is essential for poloidal mode coupling, and hence it is important for ballooning modes.

\subsection{Local equilibrium model}

From the analytic representation for the flux coordinates, (61) and (62), the elongation and the triangularity that characterize the shape of the poloidal cross section of the magnetic flux surfaces can be analytically derived in the vicinity of the magnetic axis, $\xi \ll 1$. Equation (61) is expanded up to the second order in $\xi$ as

$$
\bar{x} \simeq \bar{\Delta}_{s}+\frac{1-\bar{\Delta}_{s}^{2}}{\sqrt{1+3 \bar{\Delta}_{s}^{2}}} \xi \cos \Theta-\frac{\bar{\Delta}_{s}\left(1-\bar{\Delta}_{s}^{2}\right)^{2}}{\left(1+3 \bar{\Delta}_{s}^{2}\right)^{2}}(\xi \cos \Theta)^{2} .
$$

Substituting (73) into (62) and expanding up to the second order in $\xi$, we obtain

$$
\bar{z} \simeq \sqrt{1-\bar{\Delta}_{s}^{2}}\left(1-\frac{\bar{\Delta}_{s}}{\sqrt{1+3 \bar{\Delta}_{s}^{2}}} \xi \cos \Theta\right) \xi \sin \Theta .
$$

From (73) and (74), taking the terms up to the first order in $\xi$, the elongation $\kappa_{0}$ is obtained from

$$
\left(\bar{x}-\bar{\Delta}_{s}\right)^{2}+\frac{\bar{z}^{2}}{\kappa_{0}^{2}} \simeq \bar{r}_{c}^{2} \xi^{2},
$$

as

$$
\begin{aligned}
\kappa_{0}^{2} & =1+\frac{4 \bar{\Delta}_{s}^{2}}{1-\bar{\Delta}_{s}^{2}} \\
& =1+\frac{2[\nu /(1-F)]^{2}}{1+[\nu /(1-F)]^{2}+\sqrt{1+3[\nu /(1-F)]^{2}}}
\end{aligned}
$$


where

$$
\bar{r}_{c} \equiv \frac{1-\bar{\Delta}_{s}^{2}}{\sqrt{1+3 \bar{\Delta}_{s}^{2}}} .
$$

For the static MHD equilibrium, the elongation $\kappa_{0}$ coincides with that in [20] and indicates that the magnetic flux surfaces are elongated, $\kappa_{0}>1$, due to the high beta effect. Figure 8 (a) shows the dependence of $\kappa_{0}$ on the the poloidal Alfvén Mach number of the $E \times B$ poloidal flow velocity $V_{E c}$ for different models for $\nu=0$.8. The magnetic surfaces are elongated in the whole region of $V_{E c}$ with the peak of $\kappa_{0}=\sqrt{3}$ when $F=1$. The triangularity $\delta[38]$ is obtained by taking terms of (73) and (74) up to the second order in $\xi$ from the definition

$$
\delta(\xi) \equiv \frac{\bar{\Delta}_{s \xi}(\xi)-\bar{x}\left(\xi, \Theta=\Theta_{z \max }\right)}{\bar{r}_{c} \xi},
$$

where the Shafranov shift for the magnetic flux surface at $\xi$,

$$
\begin{aligned}
\bar{\Delta}_{s \xi}(\xi) & \equiv \frac{1}{2}[x(\xi, \Theta=0)+x(\xi, \Theta= \pm \pi)] \\
& \simeq \bar{\Delta}_{s}\left[1-\left(\frac{1-\bar{\Delta}_{s}^{2}}{1+3 \bar{\Delta}_{s}^{2}} \xi\right)^{2}\right]
\end{aligned}
$$

and $\Theta_{z \max }$ is obtained from

$$
\left.\frac{\partial \bar{z}}{\partial \Theta}\right|_{\Theta=\Theta_{z \max }}=0
$$

Substituting (74) into (80), we obtain

$$
\cos \Theta_{z \max } \simeq-\frac{\bar{\Delta}_{s} \xi}{\left(1+3 \bar{\Delta}_{s}^{2}\right)^{1 / 2}} .
$$

Thus, from (81), we obtain

$$
\bar{x}\left(\xi, \Theta=\Theta_{z \max }\right) \simeq \bar{\Delta}_{s}\left(1-\frac{1-\bar{\Delta}_{s}^{2}}{1+3 \bar{\Delta}_{s}^{2}} \xi^{2}\right) .
$$

Substituting (77), (79) and (82) into (78), we obtain $\delta$ as

$$
\delta(\xi) \simeq \frac{4 \bar{\Delta}_{s}^{3} \xi}{\left(1+3 \bar{\Delta}_{s}^{2}\right)^{3 / 2}} .
$$

Equation (83) shows that the triangularity $\delta$ is proportional to the flux coordinate $\xi$. The positive (negative) $\delta$ represents the D (reversed D) shape. For the static MHD equilibrium, the triangularity becomes positive due to the high-beta effect since $\bar{\Delta}_{s}>0$ for finite poloidal beta. Figure 8 (b) shows that the dependence of the triangularity $\delta$ on $V_{E c}$ is similar to that of figure 1 and indicates the enhancement of the $\mathrm{D}$ shape for the sub-Alfvénic poloidal flow and the reversed D shape for the super-Alfvénic poloidal flow as the poloidal curvature in (a) of figures 6 and 7 .

A local equilibrium model $[38,39,40,41,42,43]$ is constructed from the shaping factors $\bar{\Delta}_{s \xi}, \bar{r}_{c}, \kappa_{0}$ and $\delta$, derived above, as

$$
\bar{x}(\xi, \theta)=\bar{\Delta}_{s \xi}(\xi)+\bar{r}_{c} \xi\left[\cos \theta-\delta(\xi) \sin ^{2} \theta\right],
$$




$$
\bar{z}(\xi, \theta)=\kappa_{0} \bar{r}_{c} \xi \sin \theta .
$$

This is an approximation of the analytic solution (47) up to the second order in $\xi$. By the relation,

$$
\theta \simeq \Theta+\cos \Theta_{z \max } \sin \Theta
$$

(73) and (74) are reproduced from (84) and (85). Figures 9 and 10 show the magnetic flux coordinates for the local equilibrium model corresponding to figures 4 and 5, respectively. The $\xi$ coordinates for figures 9 and 10 agree well with those for 4 and 5 for small $\xi$. Regarding the poloidal coordinates, $\theta= \pm \pi / 2$ corresponds to $\Theta=\Theta_{z \max }$, which can be shown from (86) as well as from (85), while $\Theta= \pm \pi / 2$ corresponds to $\bar{x}=\bar{\Delta}_{s}$. From (84) and (85), by using the relations in Appendix for $\vartheta=\theta$, the normal curvature $\bar{\kappa}_{n}$ and $D$ is obtained as

$$
\begin{aligned}
\bar{\kappa}_{n} \simeq & -\frac{\varepsilon}{\kappa_{0}}\left(1+\frac{\bar{\Delta}_{s \xi}^{\prime}}{\bar{r}_{c}} \cos \theta\right)^{-1}\left\{\left(\frac{\bar{\Delta}_{s \xi}^{\prime}}{\bar{r}_{c}}+\cos \theta-2 \delta \sin ^{2} \theta\right)\right. \\
& \times\left[1+\left(\kappa_{0}^{2}-1\right) \cos ^{2} \theta+4 \delta \sin ^{2} \theta \cos \theta(1+\delta \cos \theta)\right]^{1 / 2} \\
& +\sin ^{2} \theta(1+2 \delta \cos \theta) \\
& \times\left\{\left(\kappa_{0}^{2}-1\right) \cos \theta-(1+2 \delta \cos \theta)\left[\frac{\bar{\Delta}_{s \xi}^{\prime}}{\bar{r}_{c}}+2 \delta\left(2 \cos ^{2} \theta-1\right)\right]\right\} \\
& \left.\times\left[1+\left(\kappa_{0}^{2}-1\right) \cos ^{2} \theta+4 \delta \sin ^{2} \theta \cos \theta(1+\delta \cos \theta)\right]^{-1 / 2}\right\}, \\
D= & \kappa_{0} \bar{r}_{c}^{2} \xi\left(1+\frac{\bar{\Delta}_{s \xi}^{\prime}}{\bar{r}_{c}} \cos \theta\right),
\end{aligned}
$$

where

$$
\frac{\bar{\Delta}_{s \xi}^{\prime}(\xi)}{\bar{r}_{c}}=-\frac{2 \bar{\Delta}_{s}\left(1-\bar{\Delta}_{s}^{2}\right)}{\left(1+3 \bar{\Delta}_{s}^{2}\right)^{3 / 2}} \xi,
$$

and the prime denotes the derivative with respect to $\xi$. From (83), we have used the relation

$$
(\xi \delta)^{\prime}=2 \delta .
$$

When $|\delta| \ll 1$ as shown in Fig. 8 (b), the normal curvature (87) is expanded with respect to $\delta$ up to the first order as

$$
\begin{aligned}
\bar{\kappa}_{n} \simeq & -\frac{\varepsilon}{\kappa_{0}} \sqrt{1+\left(\kappa_{0}^{2}-1\right) \cos ^{2} \theta}\left\{\frac{\bar{\Delta}_{s \xi}^{\prime}}{\bar{r}_{c}}+\cos \theta\right. \\
& +2 \delta \sin ^{2} \theta\left[-1+\frac{\cos \theta}{1+\left(\kappa_{0}^{2}-1\right) \cos ^{2} \theta}\left(\frac{\bar{\Delta}_{s \xi}^{\prime}}{\bar{r}_{c}}+\cos \theta\right)\right] \\
& +\frac{\sin ^{2} \theta}{1+\left(\kappa_{0}^{2}-1\right) \cos ^{2} \theta}\left\{\left(\kappa_{0}^{2}-1\right) \cos \theta-\frac{\bar{\Delta}_{s \xi}^{\prime}}{\bar{r}_{c}}\right.
\end{aligned}
$$




$$
\begin{aligned}
& +2 \delta\left\{\left(\kappa_{0}^{2}-1\right) \cos ^{2} \theta\left[1-\frac{\sin ^{2} \theta}{1+\left(\kappa_{0}^{2}-1\right) \cos ^{2} \theta}\right]\right. \\
& \left.\left.\left.-2 \cos ^{2} \theta+1-\frac{2 \bar{\Delta}_{s \xi}^{\prime}}{\bar{r}_{c}} \cos \theta\left(1+\frac{\sin ^{2} \theta}{1+\left(\kappa_{0}^{2}-1\right) \cos ^{2} \theta}\right)\right\}\right\}\right\} \\
& \times\left(1+\frac{\bar{\Delta}_{s \xi}^{\prime}}{\bar{r}_{c}} \cos \theta\right)^{-1} .
\end{aligned}
$$

Figures 11 and 12 show the poloidal profiles of (a) the normal curvature $\bar{\kappa}_{n}$ and (b) 2D poloidal Jacobian $\bar{D}$, obtained from (91) and (88), corresponding to (b) and (d) of figures 6 and 7, respectively. Since the definitions of $\Theta$ and $\theta$ are different, figures 6 (b) and 11 (a), and figures 7 (b) and 12 (a) are qualitatively different. However, these differences will be compensated with the 2D poloidal Jacobian when the flux surface is calculated.

Substituting (91) and (88) into (A.9) for $\vartheta=\theta$, the flux surface average of the normal curvature is obtained as

$$
\begin{aligned}
\left\langle\bar{\kappa}_{n}\right\rangle(\xi) \simeq & -\frac{2 \varepsilon}{\pi} \frac{1}{\kappa_{0}^{2}-1}\left\{\frac{\bar{\Delta}_{s \xi}^{\prime}(\xi)}{\bar{r}_{c}}\left[\kappa_{0}^{2} E\left(\frac{\kappa_{0}^{2}-1}{\kappa_{0}^{2}}\right)-K\left(\frac{\kappa_{0}^{2}-1}{\kappa_{0}^{2}}\right)\right]\right. \\
& \left.+\frac{2 \delta(\xi)}{\kappa_{0}^{2}-1}\left[2 \kappa_{0}^{2} E\left(\frac{\kappa_{0}^{2}-1}{\kappa_{0}^{2}}\right)-\left(\kappa_{0}^{2}+1\right) K\left(\frac{\kappa_{0}^{2}-1}{\kappa_{0}^{2}}\right)\right]\right\},
\end{aligned}
$$

where $K$ and $E$ are the complete elliptic integrals of the first and second kinds, respectively,

$$
\begin{aligned}
& K(m)=\int_{0}^{\pi / 2}{\sqrt{1-m \sin ^{2} \theta}}^{-1} \mathrm{~d} \theta, \\
& E(m)=\int_{0}^{\pi / 2} \sqrt{1-m \sin ^{2} \theta} \mathrm{d} \theta .
\end{aligned}
$$

Equation (92) shows that the non-circularity of the poloidal cross-section of the magnetic flux surface, $\kappa_{0}$ and $\delta$, as well as the Shafranov shift for the flux surfaces, $\bar{\Delta}_{s \xi}$, contributes to the flux surface average of the normal curvature. Figures 13 and 14 show the radial profiles of the flux surface average of the normal curvature $\left\langle\bar{\kappa}_{n}\right\rangle$ for the MHD and FLR+TF models, respectively. The lines for the local equilibrium model, (92), show good agreement with the curves for the numerical integration in $(\xi, \Theta)$ with $(61)$ and (62) for small $\xi$. In figure 13, $\left\langle\bar{\kappa}_{n}\right\rangle$ for the static MHD equilibrium, $V_{E c}=0$, is positive, which means good curvature on average. This result agrees with the fact that positive $\kappa_{0}^{2}-1$ and $\delta$ have stabilizing influence [40] since, as shown in figure 8, both of $\kappa_{0}^{2}-1$ and $\delta$ are positive for the static equilibrium. For sub-Alfvénic poloidal $E \times B$ flow $\left|V_{E c}\right|=0.25$, $\left\langle\bar{\kappa}_{n}\right\rangle$ is slightly enhanced. For super-Alfvénic poloidal $E \times B$ flow $\left|V_{E c}\right|=1.5,\left\langle\bar{\kappa}_{n}\right\rangle$ is negative, which means bad curvature on average. Figure 14 shows the modification due to the diamagnetic effect for the FLR+TF model. The flux surface average of the normal curvature also depends on the sign of $V_{E c}$. 
Equation (92) is further simplified in the limit $\kappa_{0}^{2} \rightarrow 1$, which is justified for $\left|V_{E c}\right| \gg 1$ as in Fig. 8 (a), as

$$
\begin{aligned}
\left\langle\bar{\kappa}_{n}\right\rangle(\xi) & \simeq \frac{\varepsilon}{4}\left[-\frac{2 \bar{\Delta}_{s \xi}^{\prime}(\xi)}{\bar{r}_{c}}+\delta(\xi)\right] \\
& =\frac{\varepsilon \bar{\Delta}_{s} \xi}{\left(1+3 \bar{\Delta}_{s}^{2}\right)^{3 / 2}} .
\end{aligned}
$$

For stability analysis, the reduced two-fluid MHD equations with FLR [11] should be exploited and linearized with perturbations around the equilibria. In addition to the modification of the magnetic curvature, other additional terms due to sheared flow modify the stability. This will be studied in future.

\section{Numerical solutions}

In this section, we present numerical solutions of (43). We developed a code with the finite element method $[44,45,46]$. We assume up-down symmetry for the poloidal crosssection. We divide the plasma region into 4-node rectangular isoparametric elements with equal lengths in $\bar{r}$ from the geometric center, $\bar{r}=0$, and $\theta$ in the quasi-toroidal coordinates. The elements around the center, consequently, become triangular. The area integration in each element is obtained from the $2 \times 2$ Gaussian quadrature. Since the GS equation is nonlinear in general, it is solved iteratively. We adopt analytic solution for MHD static equilibrium as an initial guess of $\bar{\psi}_{1}$. The initial guess of $\bar{\psi}_{1}$ is substituted into the right-hand side of (43) to find the new $\bar{\psi}_{1}$ that is used as a guess in the next step. The norm of the error vector is calculated from the differences between both sides of (43) at each grid point except for the points at the boundary, The iteration continues until the norm of the error vector becomes less than $10^{-8}$.

We, first, solve (43) for $\alpha=1$ and $\kappa=0$, (45), numerically and benchmark with its analytic solution (47). In this case, the GS equation is linear and the solution is directly obtained to satisfy the norm of the error vector less than $10^{-8}$ without iteration. We define the numerical error from the analytic solution as

$$
\delta_{e r r}^{2}=\frac{\sum_{i=1}^{N(N+1)}\left[\bar{\psi}_{1 i}-\bar{\psi}_{1}\left(\bar{r}_{i}, \theta_{i}\right)\right]^{2}}{\sum_{i=1}^{N(N+1)} \bar{\psi}_{1 i}^{2}},
$$

where $\bar{\psi}_{1 i}$ and $\bar{\psi}_{1}\left(\bar{r}_{i}, \theta_{i}\right)$ are, respectively, the numerical and analytic solutions at the $i$-th grid point except for the points at the boundary. Figure 15 shows the dependence of the error $\delta_{e r r}$ on the number of grids $N \times N$ for the FLR+TF model for $\nu=0.8$ and $V_{E c}=-0.25$. The error $\delta_{\text {err }}$ decreases with an increase of the number of grids and becomes $\sim 10^{-5}$ for $500 \times 500$ grids. 
We, next, consider the more complicated case where $\alpha=3 / 2$ and $\kappa=1$. Unlike the above case, the density $\bar{n}_{0}$ is non-uniform $\bar{n}_{0} \propto \bar{\psi}_{1}$ as well as the pressure $\bar{p}_{1} \propto \bar{\psi}_{1}^{3 / 2}$. Equations (43) and (44) become

$$
\begin{aligned}
& {\left[\frac{1}{\bar{r}} \frac{\partial}{\partial \bar{r}}\left(\bar{r} \frac{\partial}{\partial \bar{r}}\right)+\frac{1}{\bar{r}^{2}} \frac{\partial^{2}}{\partial \theta^{2}}\right] \bar{\psi}_{1}=-\frac{g_{c}}{B_{p}^{2}}-\frac{3 p_{1 c} \bar{\psi}_{1}^{1 / 2} \bar{r} \cos \theta}{(1-F) B_{p}^{2}},} \\
& F=\left(V_{E c}+\frac{3}{2} \lambda_{H} V_{d c} p_{i 1 c}\right)\left[V_{E c}+\frac{3}{2}\left(\lambda_{H}-\lambda_{i}\right) V_{d c} p_{i 1 c}\right] .
\end{aligned}
$$

Figure 16 shows the radial profiles of the numerical solutions in the midplane for superAlfvénic poloidal $E \times B$ flow $\left|V_{E c}\right|=1.5$ for $\nu=0.8$ with $500 \times 500$ grids. The temperature $\bar{T}_{1}$ in figure 16 (c) is defined as $\bar{T}_{1} \equiv p_{1} / n_{0} \propto \bar{\psi}_{1}^{1 / 2}$. The solutions have been obtained by about 10 - 20 steps of the iteration. The results in figure 16 show the similar behavior to those of the analytic solution in figure 2 as follows. The magnetic axis is located in $\bar{x}<0$. Due to the diamagnetic effect, the equilibrium is asymmetric with respect to the sign of the $E \times B$ drift velocity. The results for the TF model for $V_{E c}=1.5$ is not shown in figure 16 since no solution has been found. For $\alpha=3 / 2$, if the equilibrium is beyond the beta limit and $\bar{\psi}_{1}$ becomes negative, the pressure becomes imaginary and no real solution can be found. For the FLR+TF and FLR models, solutions are found for $V_{E c}= \pm 1.5$. For $V_{E c}=-1.5$, the FLR+TF model shows moderate difference from the MHD compared to the TF model. For the FLR model, the asymmetry with respect to the sign of the $E \times B$ drift is opposite to that for the FLR+TF model.

\section{Summary}

We have solved the equations for high-beta tokamak equilibria with flow comparable to the poloidal Alfvén velocity in the reduced MHD model with two-fluid and FLR effects analytically and numerically. By using the analytic solution, we have examined the modification of the magnetic structure in detail. From the radial profile of the magnetic flux function, we have shown that the dependence of the Shafranov shift and the equilibrium limits on the poloidal beta and the poloidal Alfvén Mach number are modified by the two-fluid and FLR effects. By constructing magnetic flux coordinates and a local equilibrium model from the analytic solution, we have shown the effects of the non-circular property of the magnetic flux surfaces in the poloidal cross-section on the components of the curvature vector. We have shown the benchmark result of the numerical code with the analytic solution. We have also shown that the numerical solutions with non-uniform pressure, density and temperature profiles show similar behavior to the analytic solution. The modification of the magnetic structure may affect instabilities. By using the equilibrium solutions obtained in this study, the stability analysis based on the reduced MHD including the effects of flow, two-fluid and FLR on both perturbation and equilibrium can be studied. 


\section{Acknowledgments}

This work was performed on "Plasma Simulator" (FUJITSU FX100) of NIFS with the support and under the auspices of the NIFS Collaboration Research program (NIFS18KNXN362, NIFS18KNSS104).

\section{Appendix A. Components of the magnetic curvature}

The components of the magnetic curvature normalized with $a^{-1}$ are written in flux coordinates $(\xi, \vartheta)$ as

$$
\begin{aligned}
& \bar{\kappa}_{p}=D^{-1}\left[\frac{\partial}{\partial \xi}\left(\sqrt{g_{22}}\right)-\frac{\partial}{\partial \vartheta}\left(\frac{g_{12}}{\sqrt{g_{22}}}\right)\right], \\
& \bar{\kappa}_{\varphi} \simeq \frac{\varepsilon \sqrt{g_{22}}}{D}\left(\frac{\partial \bar{x}}{\partial \xi}-\frac{g_{12}}{g_{22}} \frac{\partial \bar{x}}{\partial \vartheta}\right), \\
& \bar{\kappa}_{n} \simeq-\bar{\kappa}_{\varphi}, \\
& \bar{\kappa}_{g} \simeq-\frac{\varepsilon}{\sqrt{g_{22}}} \frac{\partial \bar{x}}{\partial \vartheta}
\end{aligned}
$$

where

$$
\begin{aligned}
g_{12} & =\frac{\partial \bar{x}}{\partial \xi} \frac{\partial \bar{x}}{\partial \vartheta}+\frac{\partial \bar{z}}{\partial \xi} \frac{\partial \bar{z}}{\partial \vartheta}, \\
g_{22} & =\left(\frac{\partial \bar{x}}{\partial \vartheta}\right)^{2}+\left(\frac{\partial \bar{z}}{\partial \vartheta}\right)^{2}, \\
D & =\frac{\partial \bar{x}}{\partial \xi} \frac{\partial \bar{z}}{\partial \vartheta}-\frac{\partial \bar{z}}{\partial \xi} \frac{\partial \bar{x}}{\partial \vartheta} .
\end{aligned}
$$

By substituting (61) and (62), the components of the magnetic curvature, (A.1) - (A.4), are obtained analytically. The normalized 2D poloidal Jacobian is defined as

$$
\bar{D} \equiv D / \int_{-\pi}^{\pi} D d \vartheta
$$

The flux surface average of the normal curvature is

$$
\left\langle\bar{\kappa}_{n}\right\rangle(\xi)=\int_{-\pi}^{\pi} \bar{\kappa}_{n} D d \vartheta / \int_{-\pi}^{\pi} D d \vartheta
$$




\section{References}

[1] Braginskii S I 1966 Reviews of Plasma Physics Vol 1 ed M A Leontovich (New York: Consultants Bureau) p 205

[2] Ramos J J 2005 Phys. Plasmas 12052102

[3] Schnack D D, Barnes D C, Brennan D P, Hegna C C, Held E, Kim C C, Kruger S E, Pankin A Y and Sovinec C R 2006 Phys. Plasmas 13058103

[4] Roberts K V and Taylor J B 1962 Phys. Rev. Lett. 8197

[5] Ferraro N M and Jardin S C 2006 Phys. Plasmas 13092101

[6] Zhu P, Schnack D D, Ebrahimi F, Zweibel E G, Suzuki M, Hegna C C and Sovinec C R 2008 Phys. Rev. Lett. 101, 085005

[7] Ito A and Miura H 2016 Phys. Plasmas 23122123

[8] Hazeltine R D and Meiss J D 1992 Plasma Confinement (Redwood City: Addison Wesley)

[9] Aiba N 2016 Plasma Phys. Control. Fusion 58045020

[10] Hazeltine R D, Kotschenreuther M and Morrison P J 1985 Phys. Fluids 282466

[11] Ramos J J 2007 Phys. Plasmas 14052506

[12] Kamiya K, Ida K, Sakamoto Y, Matsunaga G, Kojima A, Urano H, Oyama N, Koide Y and Kamada Y 2010 Phys. Rev. Lett. 105045004

[13] Ito A, Ramos J J and Nakajima N 2008 Plasma Fusion Res. 3034

[14] Chang Z and Callen J D 1992 Phys. Fluids 41766

[15] Hameiri E 1983 Phys. Fluids 26230

[16] Ishida A, Harahap C O, Steinhauer L C and Peng Y -K M 2004 Phys. Plasmas 115297

[17] Ito A, Ramos J J and Nakajima N 2007 Phys. Plasmas 14062502

[18] Haas F A 1972 Phys. Fluids 15141

[19] Freidberg J P 1987 Ideal Magnetohydrodynamics (New York: Plenum Press)

[20] Freidberg J P 2014 Ideal MHD (Cambridge: Cambridge University Press)

[21] Guazzotto L and Betti R 2005 Phys. Plasmas 12056107

[22] Ito A and Nakajima N 2008 AIP Conf. Proc. 1069121

[23] Ito A and Nakajima N 2009 Plasma Phys. Control. Fusion 51035007

[24] Raburn D and Fukuyama A 2010 Phys. Plasmas 17122504

[25] Ito A and Nakajima N 2011 Nucl. Fusion 51123006

[26] Ito A and Nakajima N 2013 J. Phys. Soc. Japan 82064502

[27] Ito A and Nakajima N 2019 Plasma Phys. Control. Fusion 61105006

[28] Kleibergen R and Goedbloed J P 1988 Plasma Phys. Control. Fusion 301939

[29] Strauss H R 1977 Phys. Fluids 201354

[30] Shiraishi J, Ohsaki S and Yoshida Z 2005 Phys. Plasmas 12092308

[31] Ramos J J 2005 Phys. Plasmas 12, 112301

[32] Goedbloed J P, Keppens R and Poedts S 2010 Advanced Magnetohydrodynamics (Cambridge: Cambridge University Press).

[33] Landreman M and Boozer A H 2016 Phys. Plasmas 23, 032506

[34] Dewar R L, Grimm R C, Johnson J L, Frieman E A, Greene J M and Rutherford P H 1974 Phys. Fluids 17930

[35] D'Ippolito D A and Goedbloed J P 1980 Plasma Phys. 221107

[36] Kleibergen R and Goedbloed J P 1988 Plasma Phys. Control. Fusion 301961

[37] Freidberg J P and Haas F A 1974 Phys. Fluids 17440

[38] Miller R L, Chu M S, Greene J M, Lin-Liu Y R and Waltz R E 1998 Phys. Plasmas 5973

[39] Todd A M M, Manickam J, Okabayashi M, Chance M S, Grimm R C, Greene J M and Johnson J L 1979 Nucl. Fusion 19743

[40] Medvedev S Y, Kikuchi M, Villard L, Takizuka T, Diamond P, Zushi H, Nagasaki K, Duan X, Wu Y, Ivanov A A, Martynov A A, Poshekhonov Y Y, Fasoli A and Sauter O 2015 Nucl. Fusion 55 063013 
[41] Zhou D and Yu W 2011 Phys. Plasmas 18052505

[42] Yu W, Zhou D and Xiang N 2012 Phys. Plasmas 19072520

[43] Yu W, Zhou D and Xiang N 2015 Phys. Scr. 90035602

[44] Bathe K J and Wilson E L 1976 Numerical Methods in Finite Element Analysis (Englewood Cliffs: Prentice-Hall)

[45] Kerner W and Jandl O 1984 Comp. Phys. Comm. 31269

[46] Furukawa M, Nakamura Y, Hamaguchi S and Wakatani M 2000 J. Plasma Fusion Res. 76937 

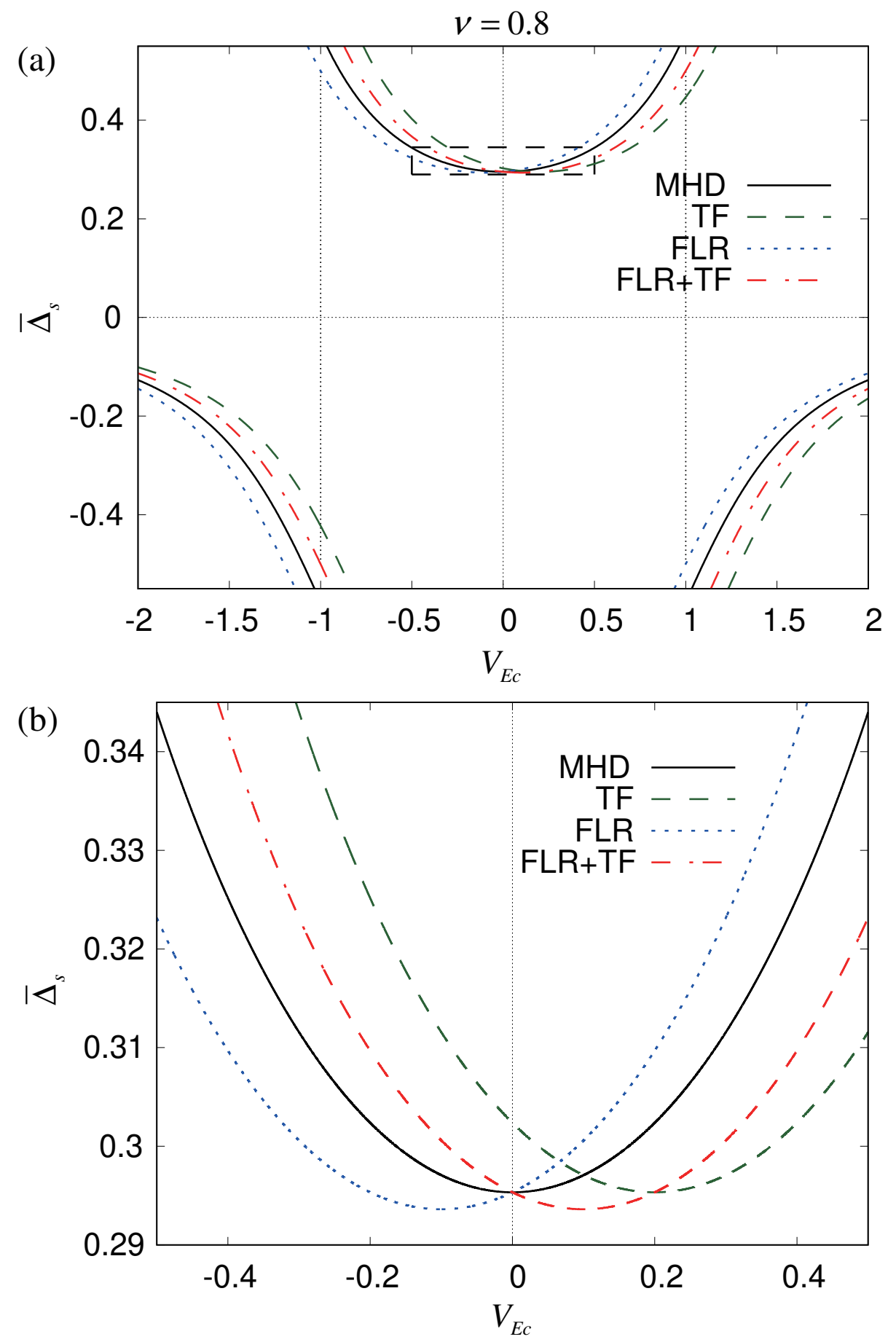

Figure 1. The Shafranov shift $\bar{\Delta}_{s}$ as a function of the poloidal Alfvén Mach number of the poloidal $E \times B$ drift $V_{E c}$ for different models for $\nu=0.8$ and $V_{d c}=-0.2$. The region around $V_{E c}=0$ in (a) is enlarged in (b). 


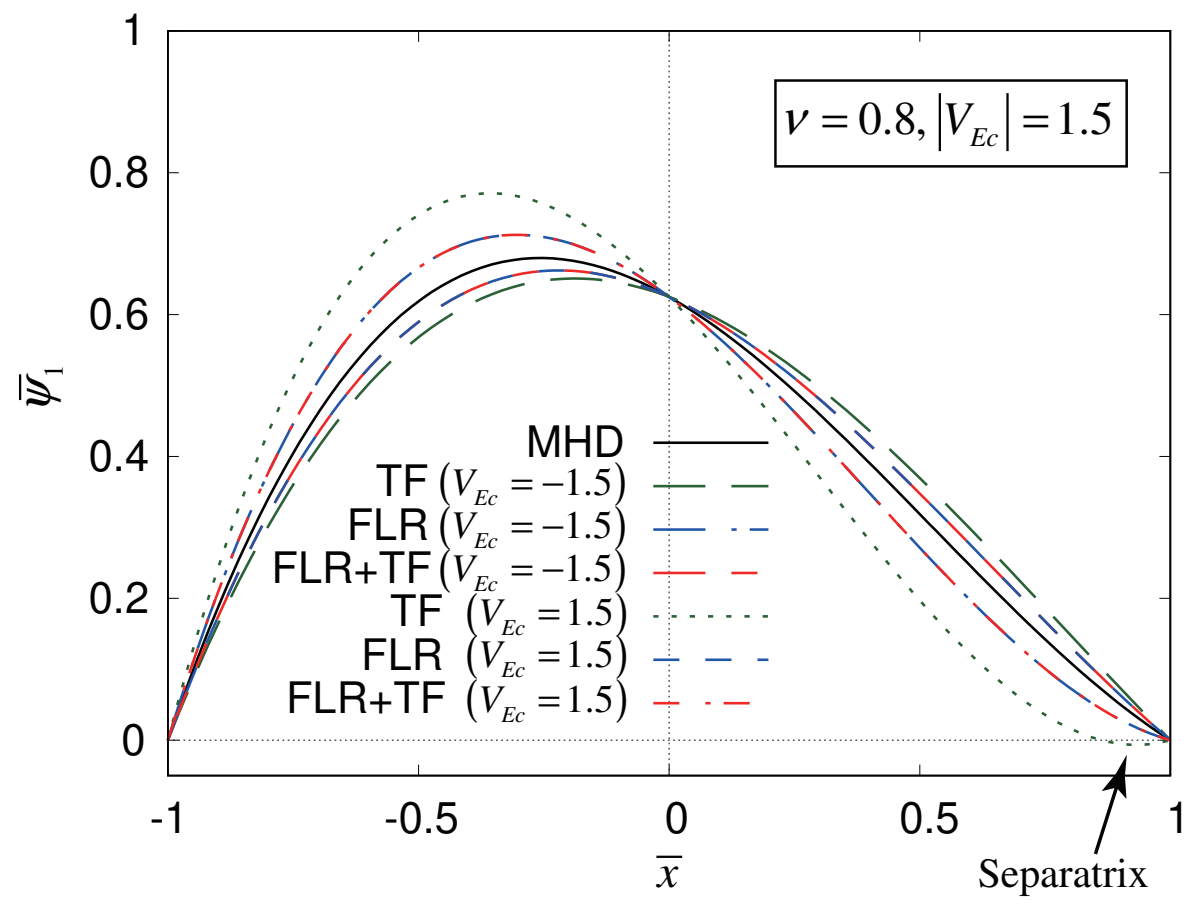

Figure 2. Radial profiles of $\bar{\psi}_{1}$ in the midplane for the different models for $\nu=0.8$, $\left|V_{E c}\right|=1.5$ and $V_{d c}=-0.2$. 

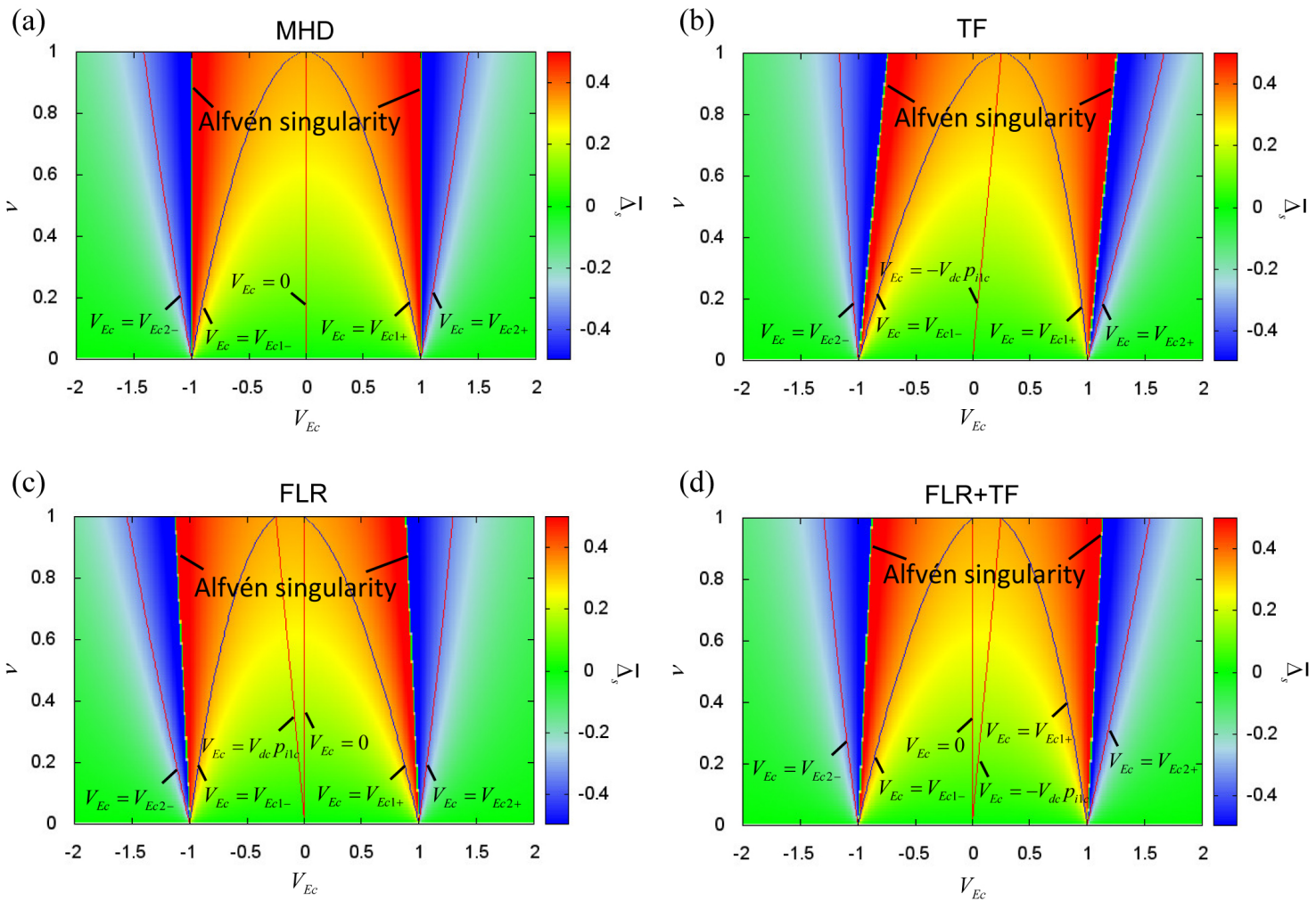

Figure 3. The limits of $E \times B$ flow, (52) and (54), on the contour map of the Shafranov shift $\bar{\Delta}_{s}$ in the $V_{E c}-\nu$ plane for the (a) MHD, (b) TF, (c) FLR and (d) FLR+TF models for $V_{d c}=-0.2$. 


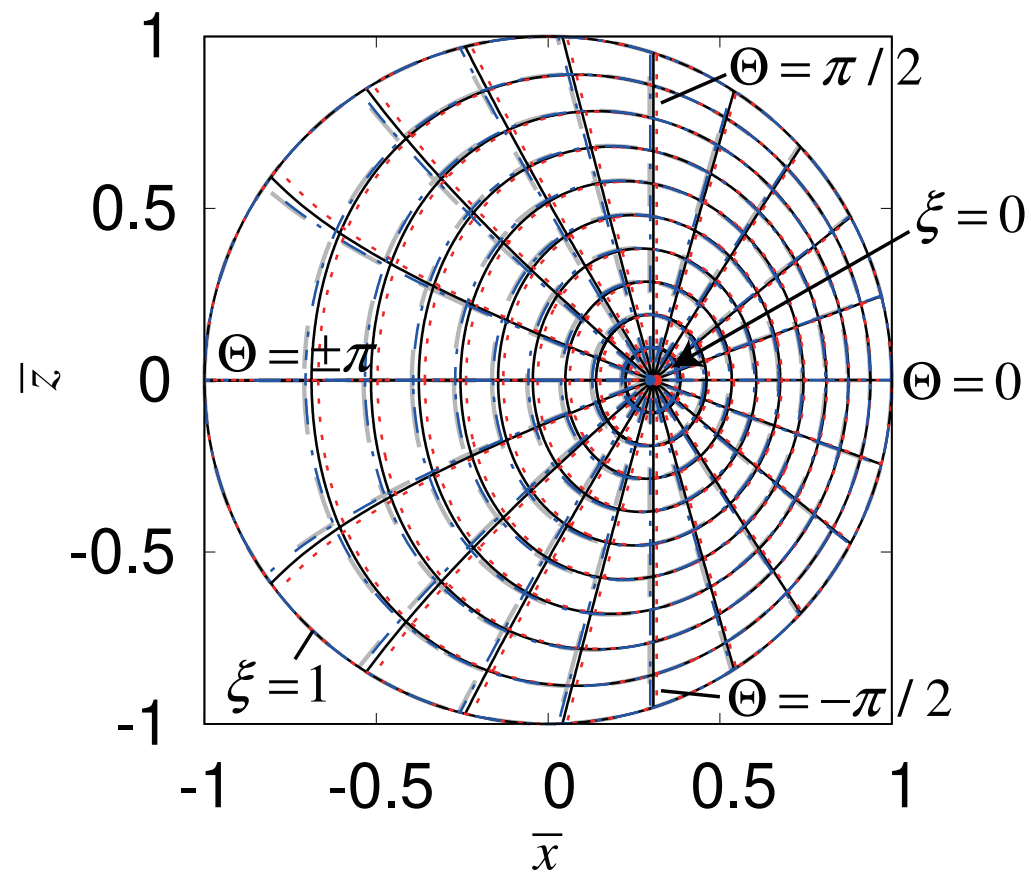

Figure 4. Magnetic flux coordinates $(\xi, \Theta)$ for $\left|V_{E c}\right|=0.25, \nu=0.8$ and $V_{d c}=-0.2$. Solid lines are for the MHD with $\left|V_{E c}\right|=0.25$. Dotted lines (red) are for the TF+FLR model with $V_{E c}=-0.25$. Dash-dotted lines (blue) are for the TF+FLR model with $V_{E c}=0.25$. Dashed lines (gray) are for the static MHD for comparison. 


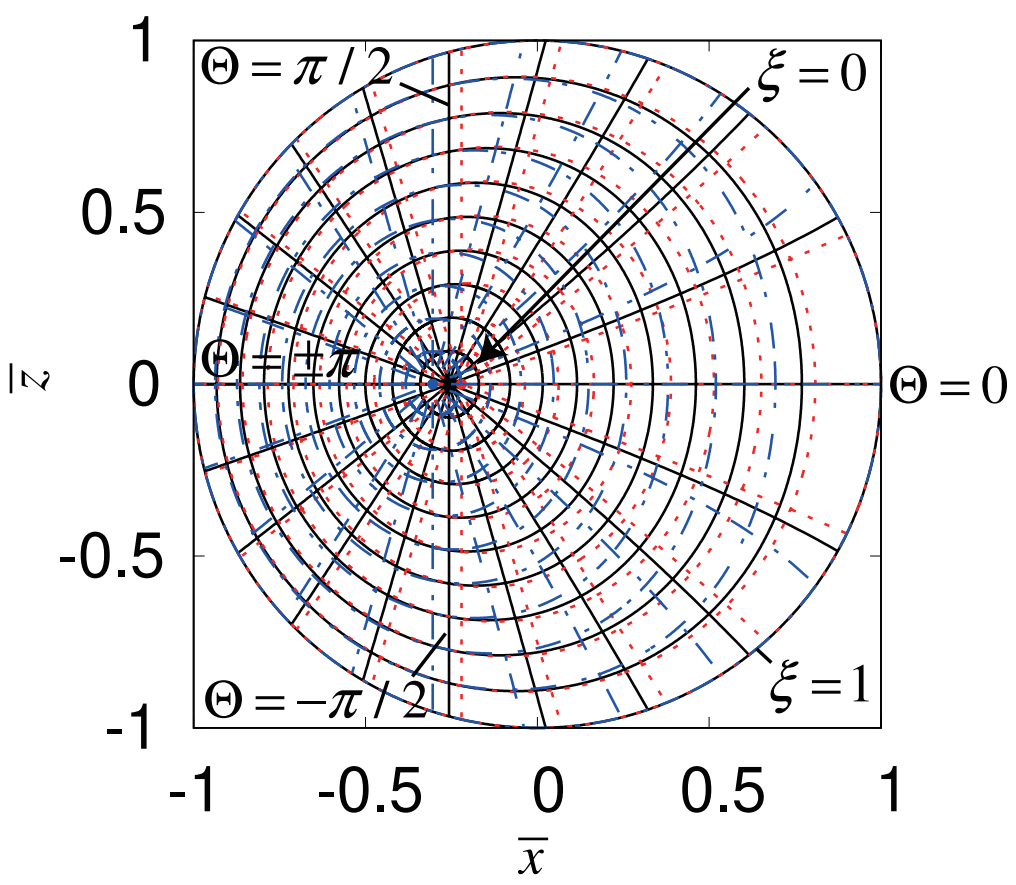

Figure 5. Magnetic flux coordinates $(\xi, \Theta)$ for $\left|V_{E c}\right|=1.5, \nu=0.8$ and $V_{d c}=-0.2$. Solid lines are for the MHD with $\left|V_{E c}\right|=1.5$. Dotted lines (red) are for the TF+FLR model with $V_{E c}=-1.5$. Dashed-dotted lines (blue) are for the TF+FLR model with $V_{E c}=1.5$. 


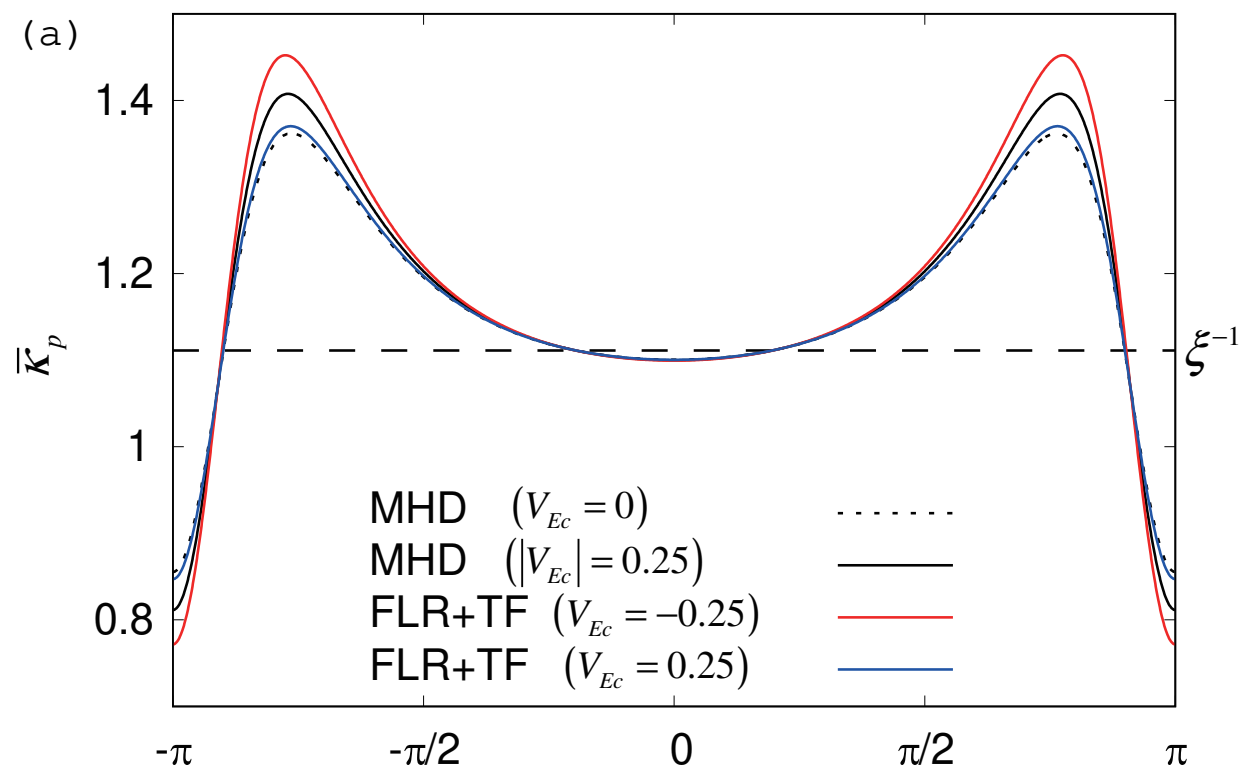

$\Theta$

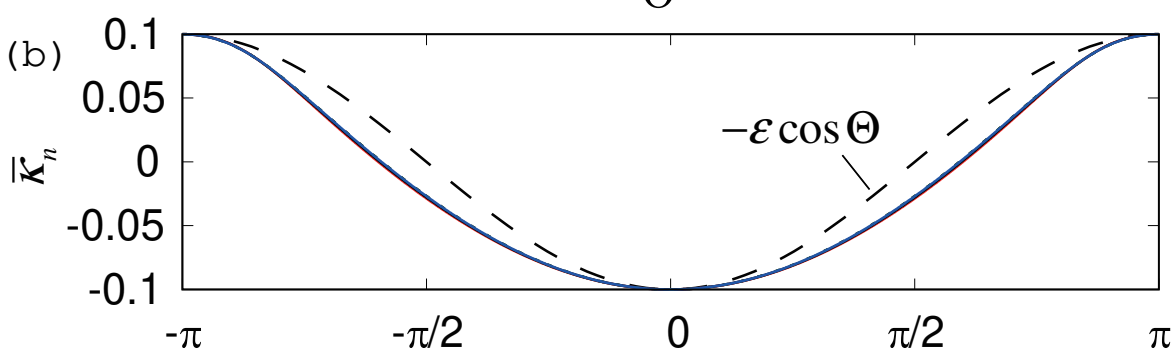

$\Theta$

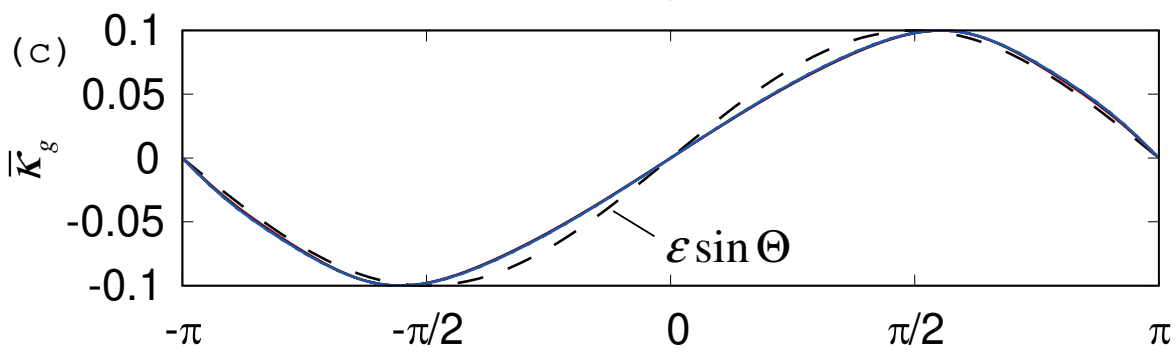

$\Theta$

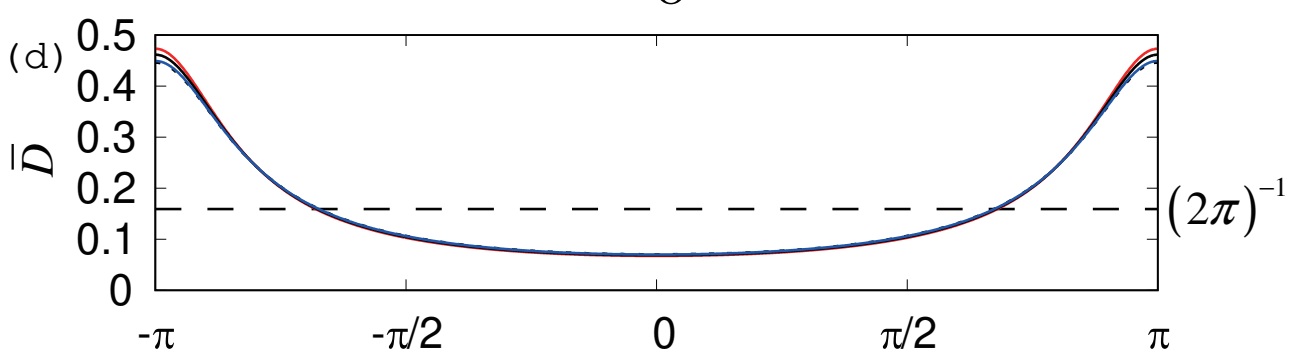

$\Theta$

Figure 6. Poloidal angle $\Theta$ profiles of (a) the poloidal curvature $\bar{\kappa}_{p}$, (b) the normal curvature $\bar{\kappa}_{n}$, (c) the geodesic curvature $\bar{\kappa}_{g}$ and (d) the 2D poloidal Jacobian $\bar{D}$ at $\xi=0.9$ for different models for $\left|V_{E c}\right|=0.25, \nu=0.8$ and $V_{d c}=-0.2$, compared to the static MHD equilibrium. 

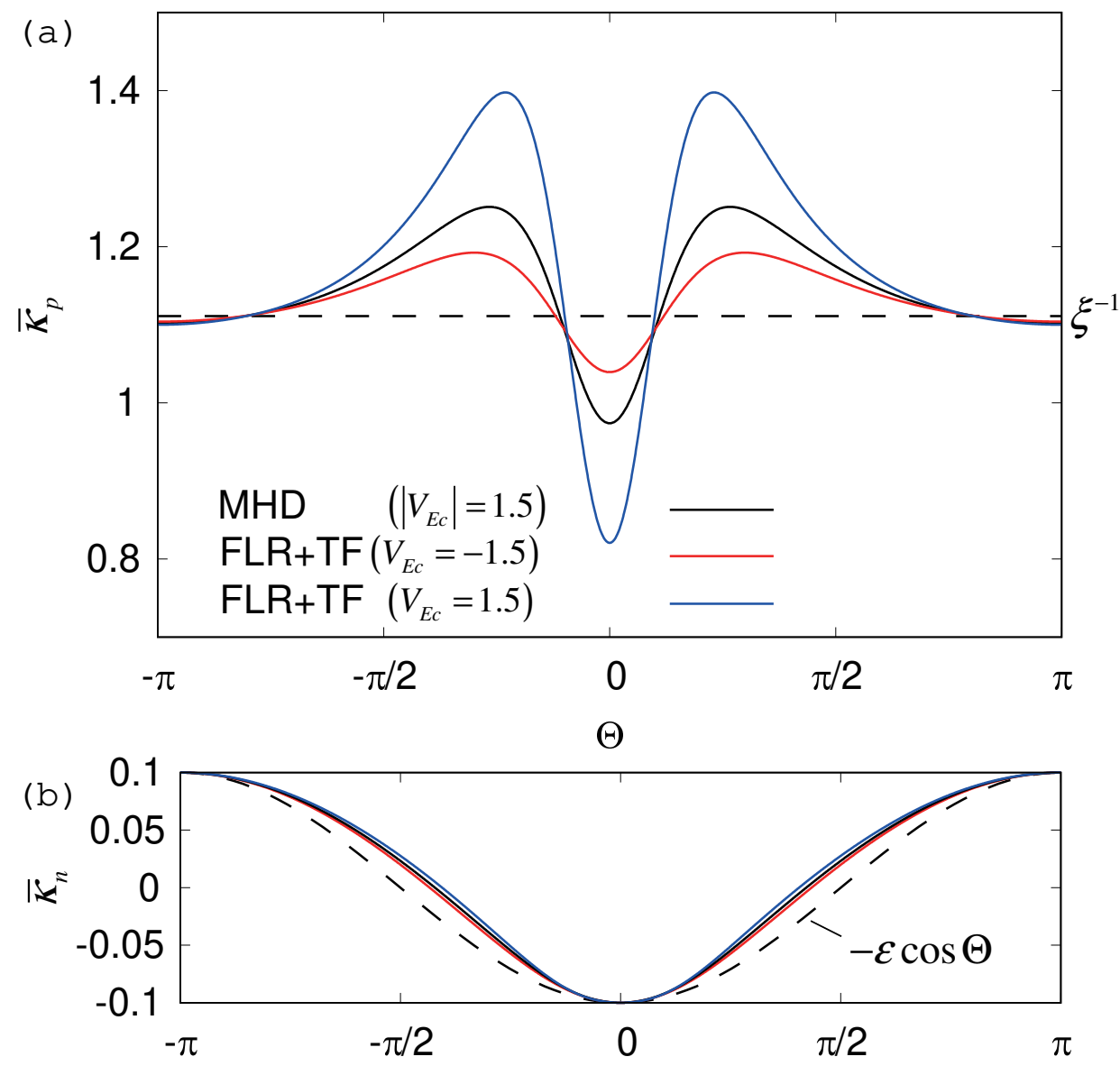

$\Theta$

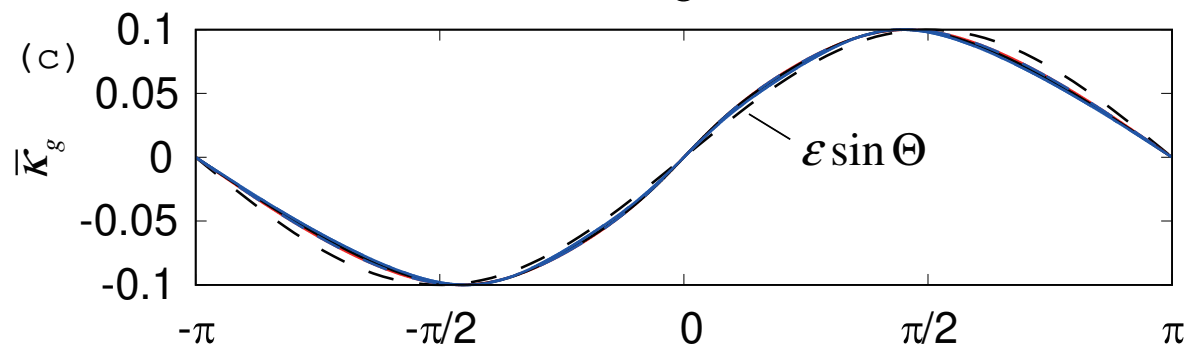

$\Theta$

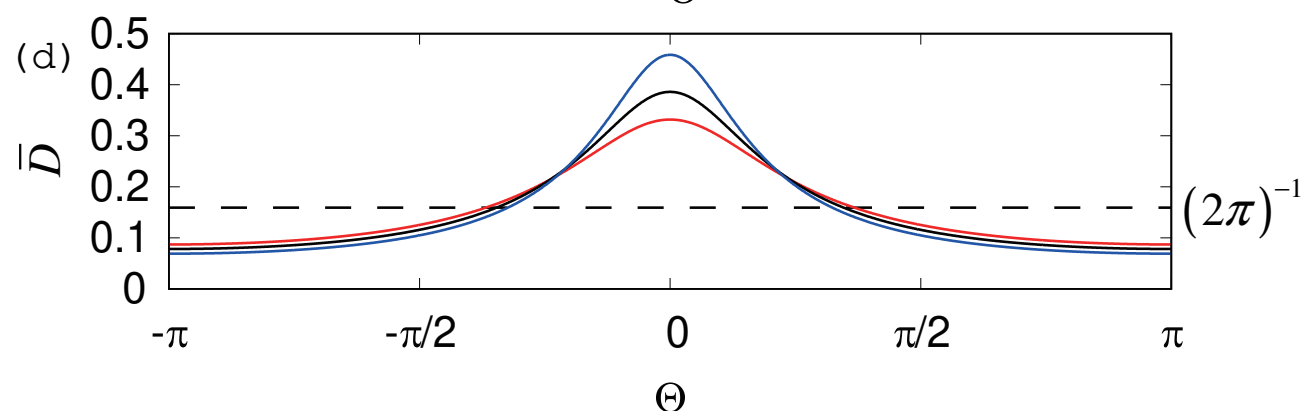

Figure 7. Poloidal angle $\Theta$ profiles of (a) the poloidal curvature $\bar{\kappa}_{p}$, (b) the normal curvature $\bar{\kappa}_{n}$, (c) the geodesic curvature $\bar{\kappa}_{g}$ and (d) the 2D poloidal Jacobian $\bar{D}$ at $\xi=0.9$ for different models for $\left|V_{E c}\right|=1.5, \nu=0.8$ and $V_{d c}=-0.2$, compared to the static MHD equilibrium. 

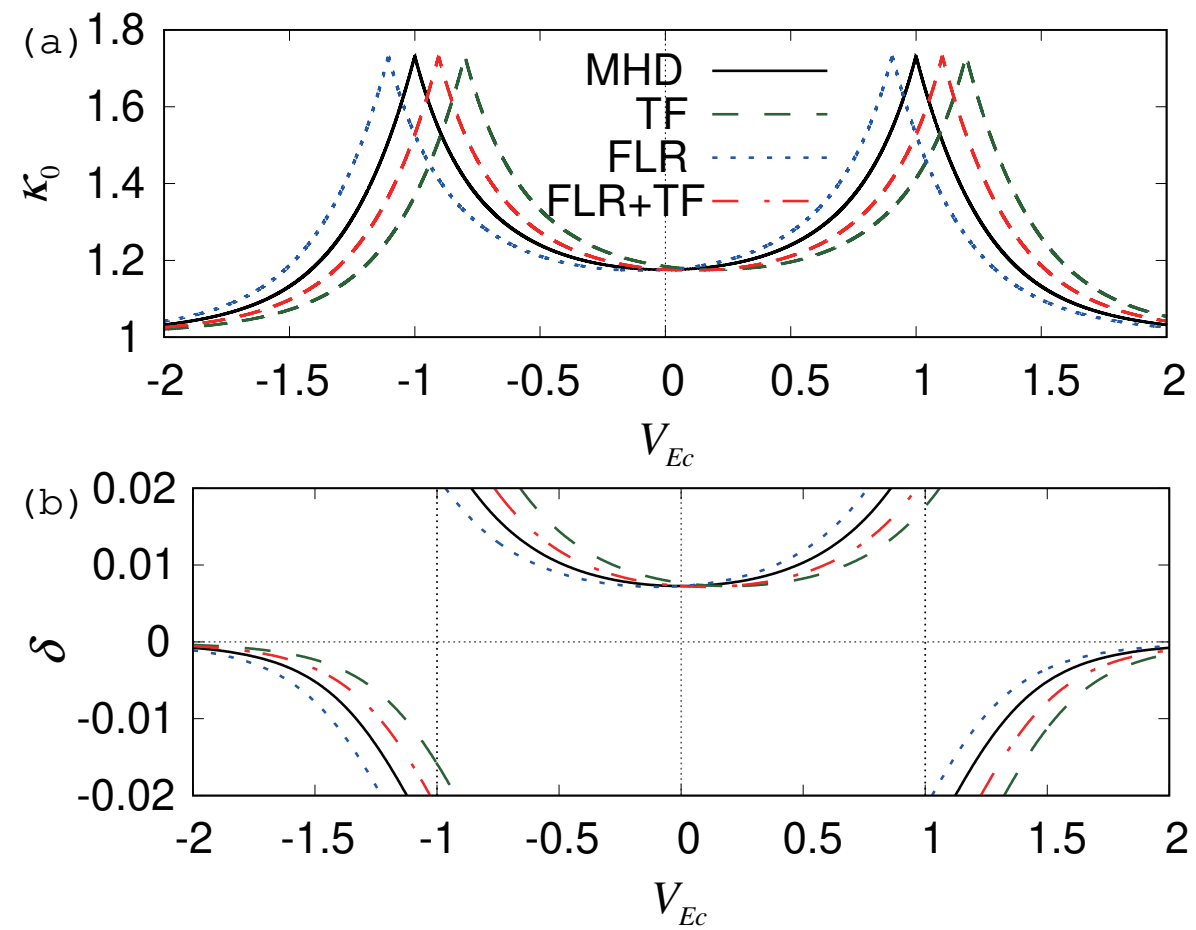

Figure 8. (a) The elongation $\kappa_{0}$ and (b) the triangularity $\delta$ at $\xi=0.1$ as a function of the poloidal Alfvén Mach number of the poloidal $E \times B$ drift $V_{E c}$ for different models for $\nu=0.8$ and $V_{d c}=-0.2$. 


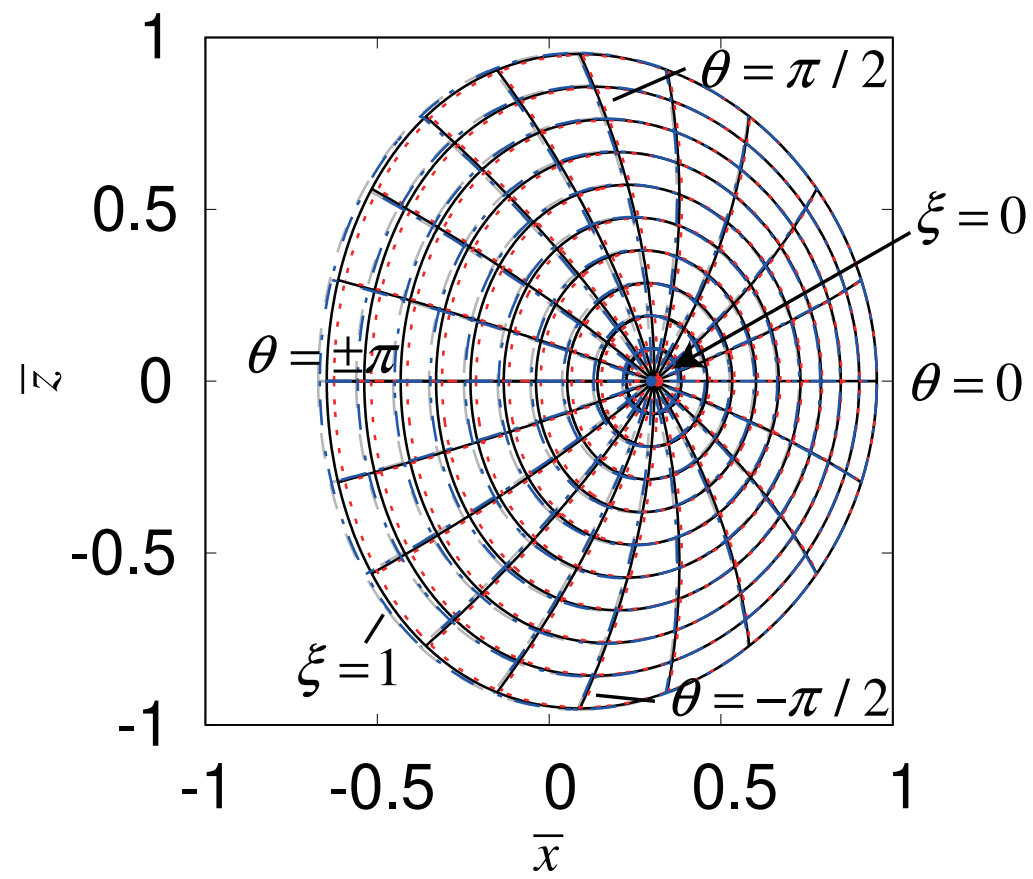

Figure 9. Magnetic flux coordinates for the local equilibrium model $(\xi, \theta)$ for $\left|V_{E c}\right|=0.25, \nu=0.8$ and $V_{d c}=-0.2$. Solid lines are for the MHD with $\left|V_{E c}\right|=0.25$. Dotted lines (red) are for the TF+FLR model with $V_{E c}=-0.25$. Dash-dotted lines (blue) are for the TF+FLR model with $V_{E c}=0.25$. Dashed lines (gray) are for the static MHD for comparison. 


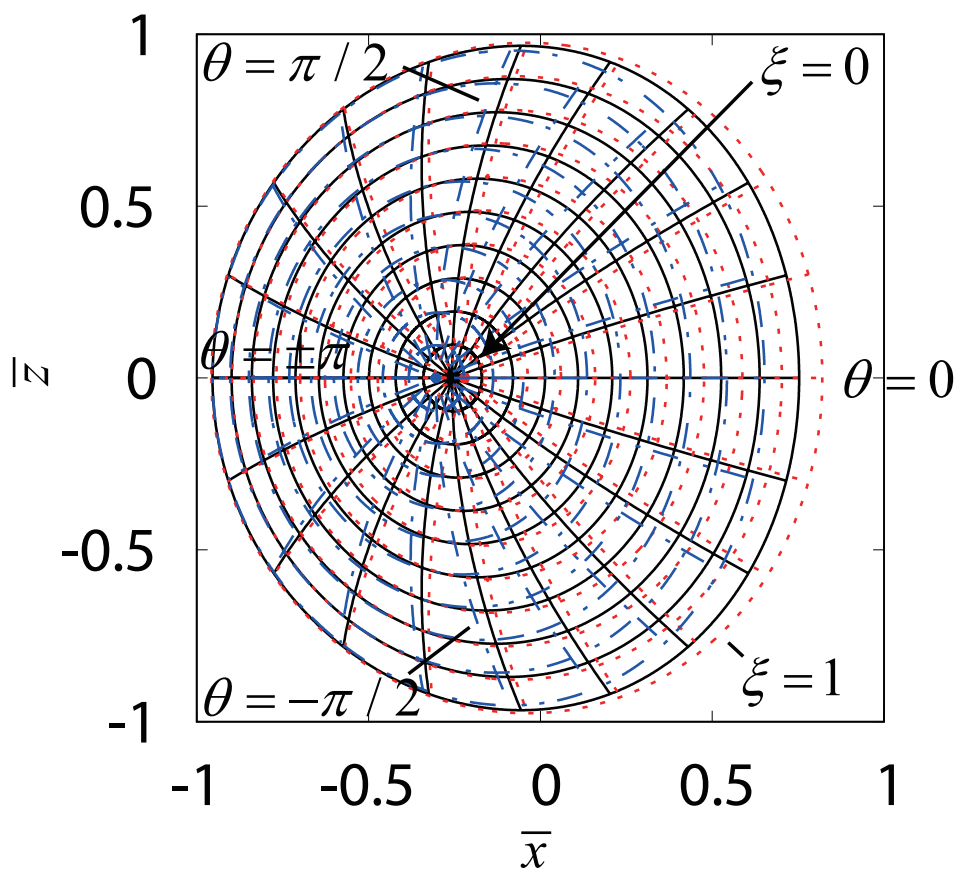

Figure 10. Magnetic flux coordinates for the local equilibrium model $(\xi, \theta)$ for $\left|V_{E c}\right|=1.5, \nu=0.8$ and $V_{d c}=-0.2$. Solid lines are for the MHD with $\left|V_{E c}\right|=1.5$. Dotted lines (red) are for the TF+FLR model with $V_{E c}=-1.5$. Dashed-dotted lines (blue) are for the TF+FLR model with $V_{E c}=1.5$. 

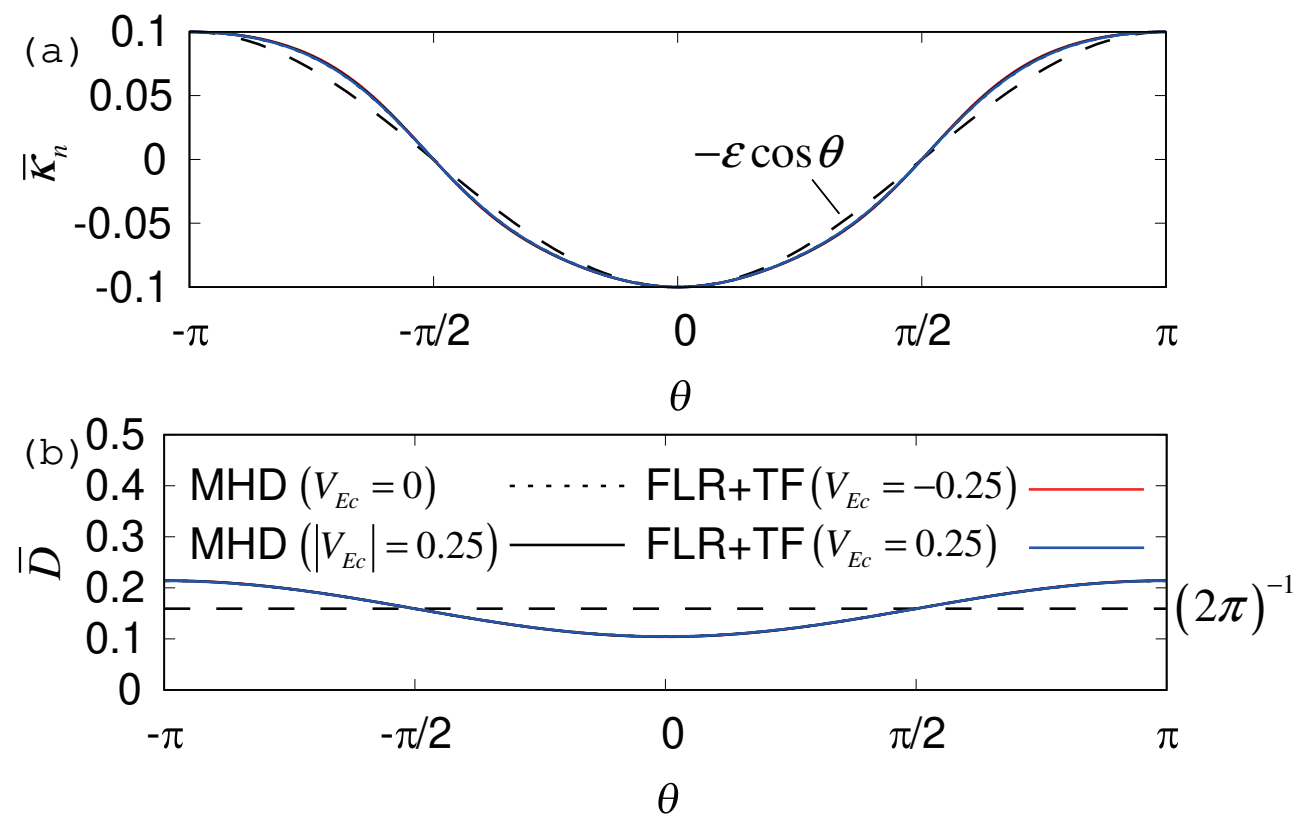

Figure 11. Poloidal angle $\theta$ profiles of (a) the normal curvature $\bar{\kappa}_{n}$ and (b) $\bar{D} \equiv D / \int_{-\pi}^{\pi} D d \theta$ at $\xi=0.9$ for different models for $\left|V_{E c}\right|=0.25, \nu=0.8$ and $V_{d c}=-0.2$, compared to the static MHD equilibrium. 

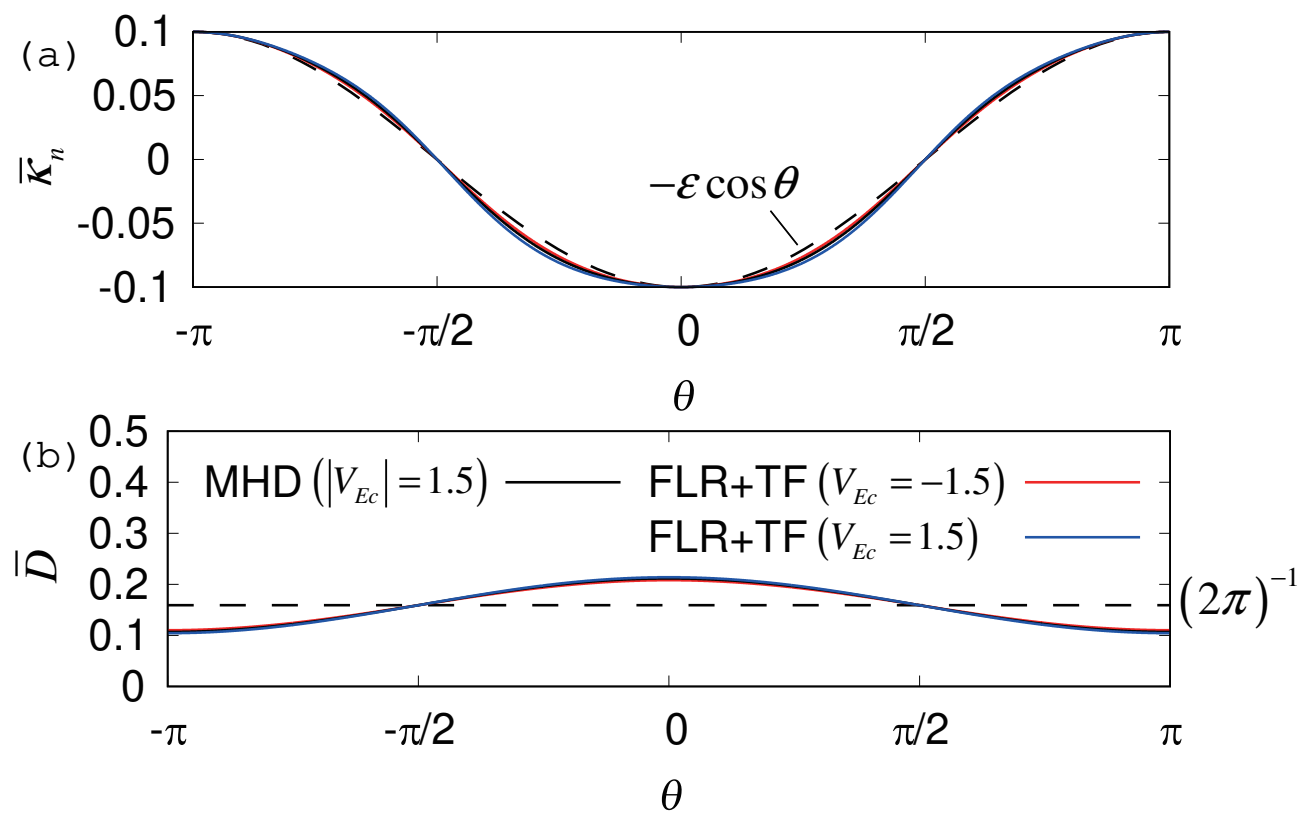

Figure 12. Poloidal angle $\theta$ profiles of (a) the poloidal curvature $\bar{\kappa}_{p}$, (b) the normal curvature $\bar{\kappa}_{n}$, (c) the geodesic curvature $\bar{\kappa}_{g}$ and (d) $\bar{D} \equiv D / \int_{-\pi}^{\pi} D d \theta$ at $\xi=0.9$ for different models for $\left|V_{E c}\right|=1.5, \nu=0.8$ and $V_{d c}=-0.2$, compared to the static MHD equilibrium. 


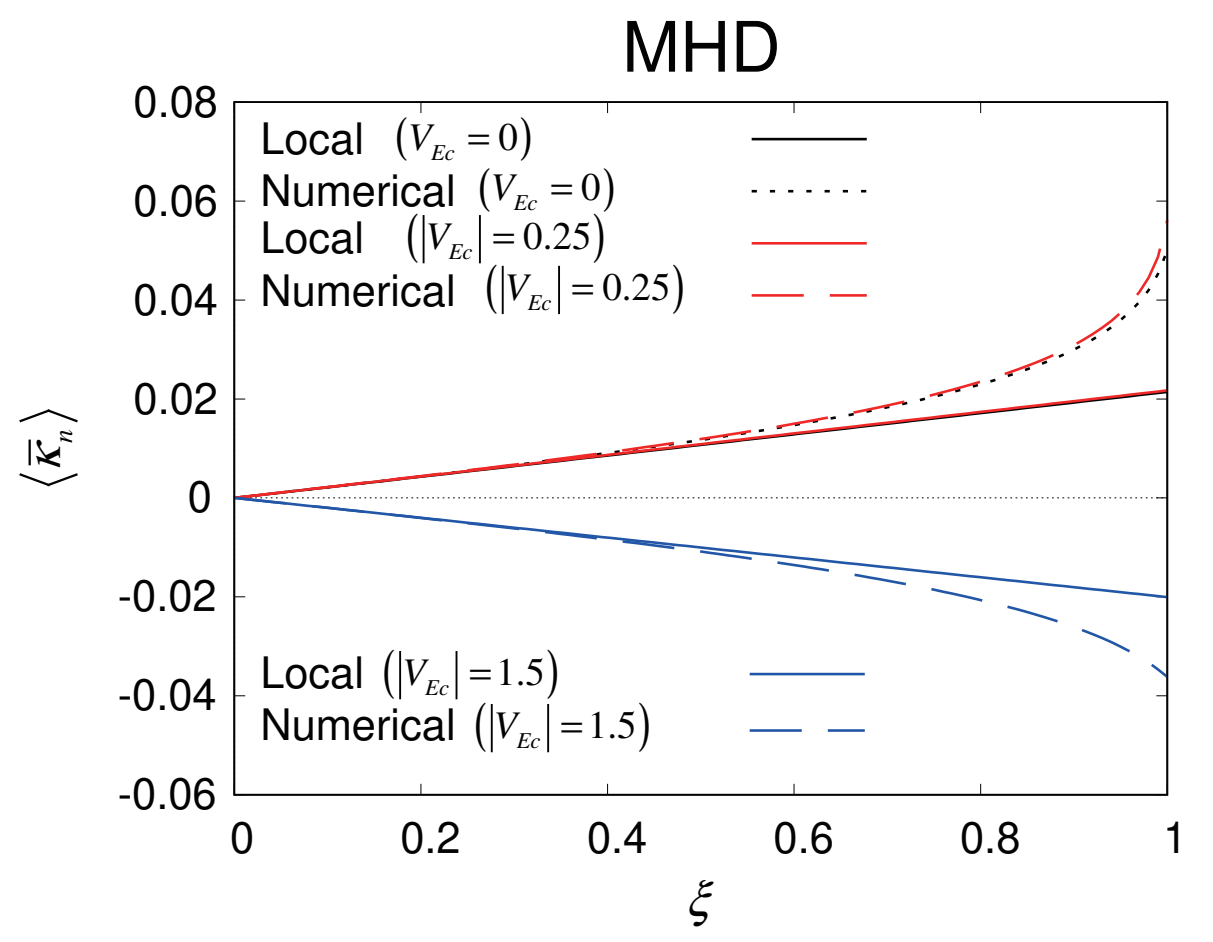

Figure 13. Radial profiles of the flux surface average of the normal curvature for the MHD model with different $V_{E c}$ obtained from the local equilibrium model (Local) and from the numerical integration in $(\xi, \Theta)$ (Numerical) for $\nu=0.8$. 


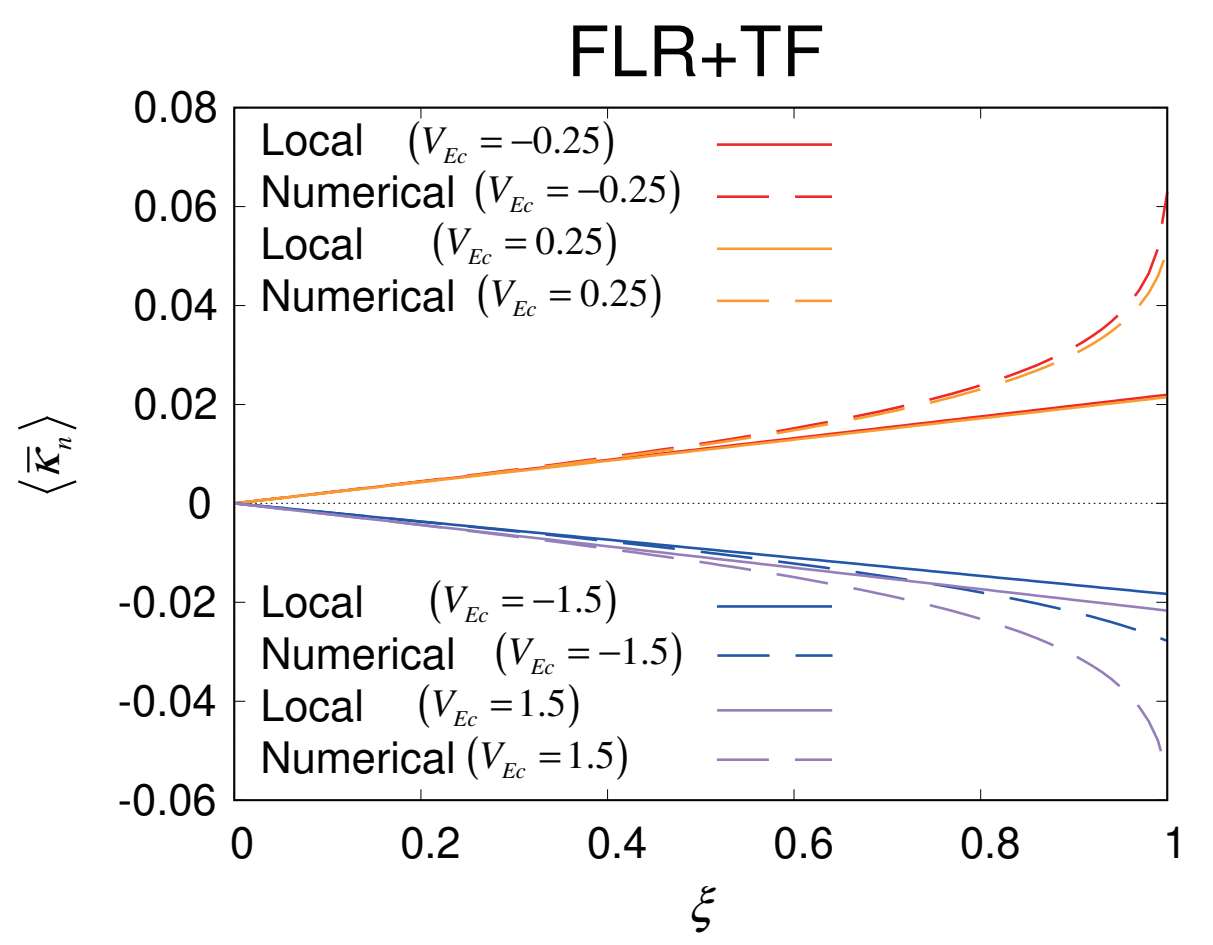

Figure 14. Radial profiles of the flux surface average of the normal curvature for the FLR+TF model with different $V_{E c}$ obtained from the local equilibrium model (Local) and from the numerical integration in $(\xi, \Theta)$ (Numerical) for $\nu=0.8$ and $V_{d c}=-0.2$. 


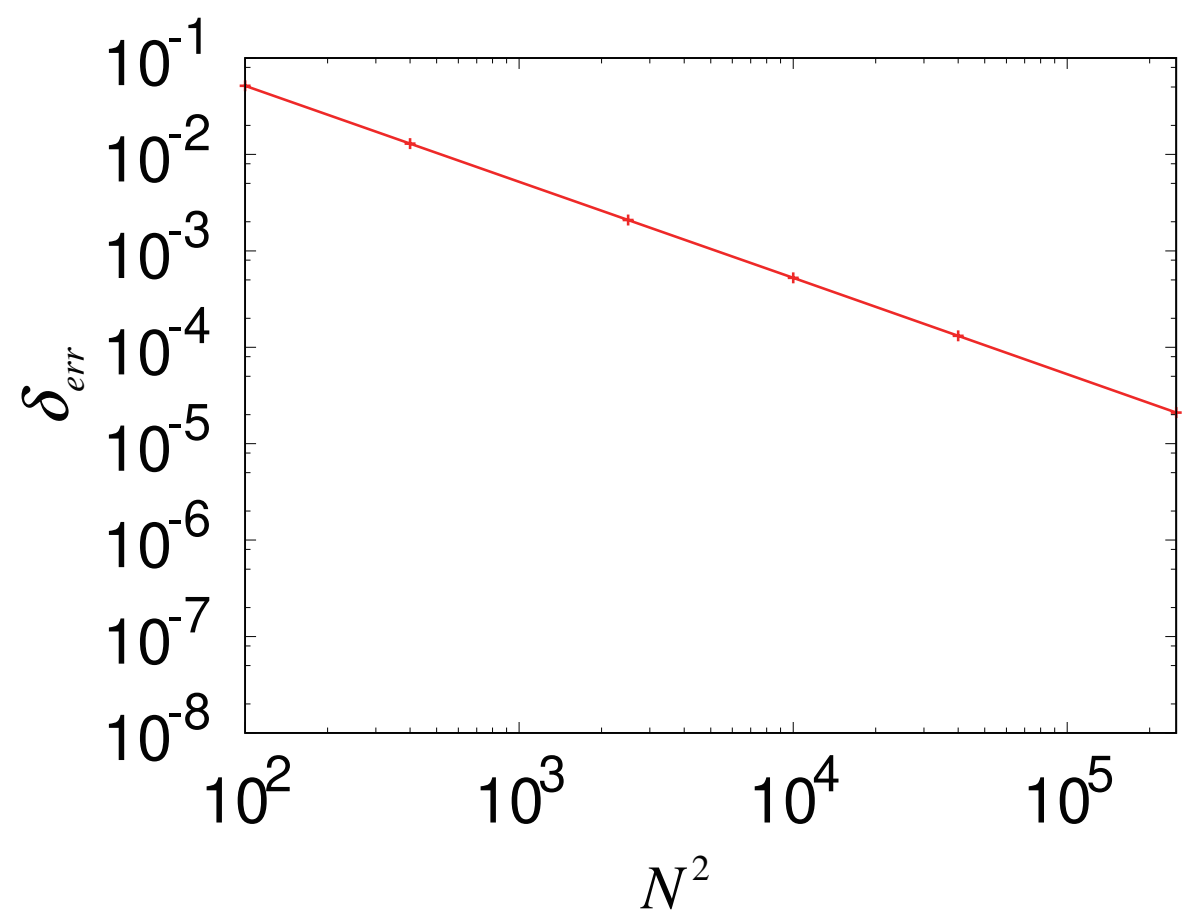

Figure 15. The numerical error from the analytic solution $\delta_{e r r}$ as a function of the number of grids. 

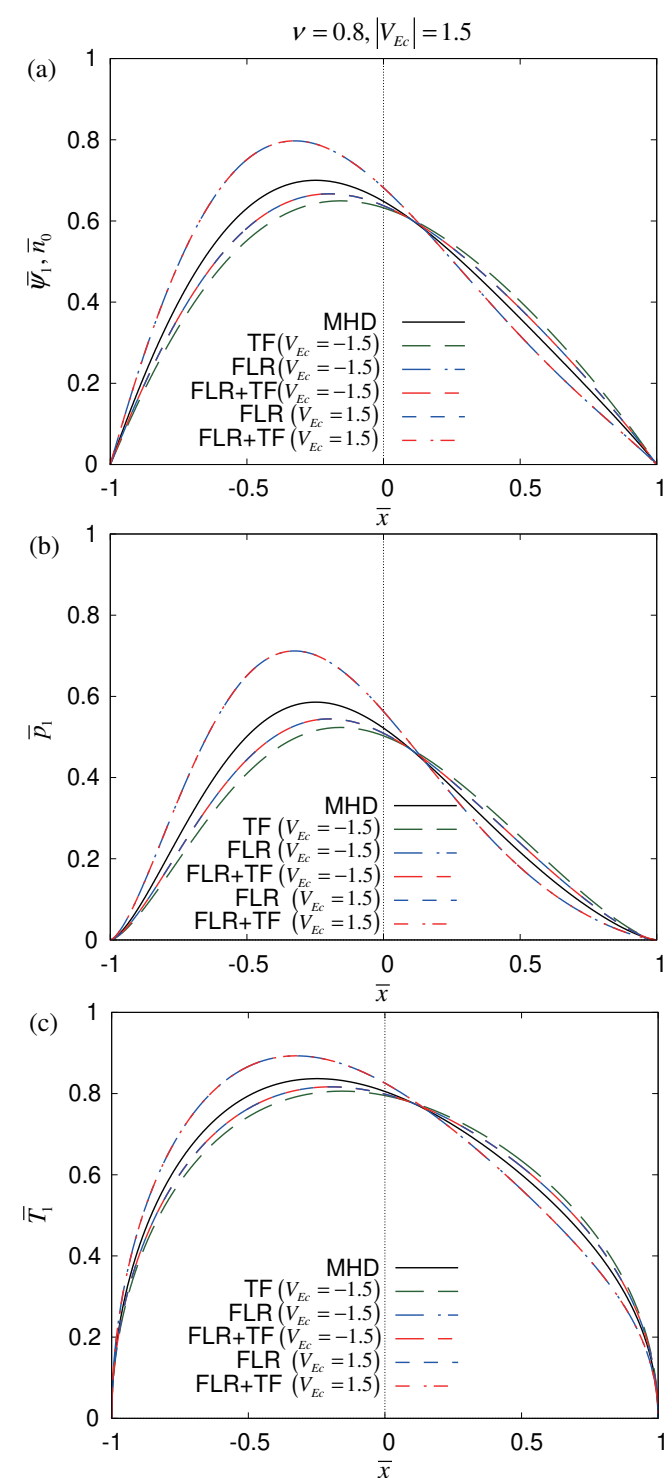

Figure 16. Numerical results of profiles in the midplane for different models for $\left|V_{E c}\right|=1.5, \nu=0.8$ and $V_{d c}=-0.2$. 